\title{
APPROXIMATE SOLUTION OF URYSOHN INTEGRAL EQUATIONS WITH NON-SMOOTH KERNELS
}

\author{
REKHA P. KULKARNI AND T.J. NIDHIN \\ Communicated by Kendall Atkinson
}

\begin{abstract}
Consider a nonlinear operator equation $x-$ $K(x)=f$, where $K$ is a Urysohn integral operator with a kernel of the type of Green's function and defined on $L^{\infty}[0,1]$. For $r \geq 0$, we choose the approximating space to be a space of discontinuous piecewise polynomials of degree $\leq r$ with respect to a quasi-uniform partition of $[0,1]$ and consider an interpolatory projection at $r+1$ Gauss points. Previous authors have proved that the orders of convergence in the collocation and the iterated collocation methods are $r+1$ and $r+2+\min \{r, 1\}$, respectively. We show that the order of convergence in the iterated modified projection method is 4 if $r=0$ and is $2 r+3$ if $r \geq 1$. This improvement in the order of convergence is achieved while retaining the size of the system of equations that needs to be solved, the same as in the case of the collocation method. Numerical results are given for specific examples.
\end{abstract}

1. Introduction. We are interested in approximate solutions of the following nonlinear operator equation

$$
x-K(x)=f,
$$

where $K$ is a Urysohn integral operator with a continuous kernel defined as follows:

$$
K(x)(s)=\int_{0}^{1} \kappa(s, t, x(t)) d t, \quad s \in[0,1], x \in L^{\infty}[0,1] .
$$

2010 AMS Mathematics subject classification. Primary 45L10, 65J15, 65R20.

Keywords and phrases. Urysohn integral operator, collocation method, Gauss points.

The first author would like to thank the Indo-French Centre for Applied Mathematics (IFCAM), Bangalore, India, for the partial support.

Received by the editors on May 26, 2015, and in revised form on January 28, 2016. 
It is assumed that the above equation has a unique solution $\varphi$, and we consider projection methods to approximate it. For $r \geq 0$, let $X_{n}$ be the space of piecewise polynomials of degree $\leq r$ with respect to a quasiuniform partition of $[0,1]$ with $n$ subintervals. Let $Q_{n}$ from $L^{\infty}[0,1]$ to $X_{n}$ be a sequence of interpolatory projections at $r+1$ Gauss points.

In the collocation method, the above equation is approximated by

$$
\varphi_{n}^{C}-Q_{n} K\left(\varphi_{n}^{C}\right)=Q_{n} f .
$$

This method has been studied extensively in research literature, see Krasnoselskii [9], Krasnoselskii et al. [10] and Krasnoselskii and Zabreiko [11].

The iterated collocation solution is defined by $\varphi_{n}^{S}=K\left(\varphi_{n}^{C}\right)+f$. It was introduced by Sloan [15] for linear integral equations, and, for nonlinear integral equations, see Atkinson and Potra [4].

In Grammont and Kulkarni [7], the following modified projection method is proposed:

$$
\varphi_{n}^{M}-K_{n}^{M}\left(\varphi_{n}^{M}\right)=f,
$$

where $K_{n}^{M}(x)=Q_{n} K(x)+K\left(Q_{n} x\right)-Q_{n} K\left(Q_{n} x\right)$. It is a generalization of the modified projection method in the linear case proposed in Kulkarni [12]. The iterated modified projection solution is defined as $\widetilde{\varphi}_{n}^{M}=K\left(\varphi_{n}^{M}\right)+f$.

If the kernel $\kappa$ is smooth, then in Grammont et al. [8], it is shown that $\left\{\varphi_{n}^{M}\right\}$ converges faster to $\varphi$ than does the sequence $\left\{\varphi_{n}^{S}\right\}$, while the size of the system of equations that needs to be solved remains the same. One iteration step used in defining the iterated modified projection solution is shown to further improve the order of convergence. In Kulkarni and Nidhin [14], asymptotic series expansions for $\varphi_{n}^{S}$ and for $\widetilde{\varphi}_{n}^{M}$ are obtained and Richardson extrapolation is used to improve the order of convergence.

In this paper, we consider a Urysohn integral operator with a Green's function type kernel. In Atkinson and Potra [4], orders of convergence of the collocation and the iterated collocation solutions for this type of kernel are obtained. These error bounds generalize the results of Chatelin and Lebbar [5] in the case of the linear integral equations. Under appropriate conditions, we show that, if $r=0$, then the orders of convergence of the modified projection and the iterated 
modified projection solutions are, respectively, 3 and 4. These orders of convergence are to be compared with the order 1 of the collocation solution and the order 2 of the iterated collocation solution. If $r \geq 1$, then we show that the order of convergence of the iterated modified projection solution is $2 r+3$, which is an improvement over the order of convergence $r+3$ of the iterated collocation solution obtained in Atkinson and Potra [4].

The paper has been arranged in the following way. In Section 2, we set the notations, state the assumptions on the kernel of the Urysohn integral operator and describe the method. In Section 3, we first prove two important results about the divided difference of $K^{\prime}(\varphi) g$, where $K^{\prime}(\varphi)$ denotes the Fréchet derivative of $K$ at $\varphi$ and $g \in C[0,1]$. This section also contains a crucial result based on the relation between the interpolatory projection at Gauss points and the orthogonal projection. Using this result, we obtain orders of convergence of certain quantities which are needed later on. In subsection 4.1, we obtain the order of convergence of the modified projection solution. Subsection 4.2 contains our main result about the order of convergence of the iterated modified projection solution. Numerical results are given in Section 5.

2. Method, notation and definitions. In this section, we set the notations and describe the method. In subsection 2.1, the Urysohn integral operator with a Green's function type kernel is defined and its Fréchet derivatives up to the order 4 are described. In subsection 2.2, the approximating space of piecewise polynomials is described. The interpolatory projection at Gauss points is defined in subsection 2.3 and some results from Chatelin and Lebbar [5] are quoted for future reference. The modified projection method and its iterative version are given in subsection 2.4, and a theorem about the existence of the modified projection solution in a neighborhood of the exact solution is stated.

2.1. Urysohn integral operator. Let $X=L^{\infty}[0,1]$, and consider a Urysohn integral operator

$$
K(x)(s)=\int_{0}^{1} \kappa(s, t, x(t)) d t, \quad s \in[0,1], x \in X
$$


where the kernel $\kappa(s, t, u)$ is a real-valued continuous function. The domain of the kernel $\kappa$ is denoted by $\Psi=[0,1] \times[0,1] \times \mathbb{R}$. Divide $\Psi$ into two parts:

$$
\Psi_{1}=\{(s, t, u): 0 \leq t \leq s \leq 1, u \in \mathbb{R}\},
$$

and

$$
\Psi_{2}=\{(s, t, u): 0 \leq s \leq t \leq 1, u \in \mathbb{R}\} .
$$

For a function $\xi(s, t, u)$ defined on an open subset $S \subset \mathbb{R}^{3}$, and for non-negative integers $i, j$ and $k$, we introduce the following notation:

$$
\left(D^{(i, j, k)} \xi\right)(s, t, u)=\frac{\partial^{i+j+k} \xi(s, t, u)}{\partial s^{i} \partial t^{j} \partial u^{k}}, \quad(s, t, u) \in S .
$$

Let $\alpha \geq 1$ be an integer. We say that $\xi \in C^{\alpha}\left(\Psi_{1}\right)$ provided the following conditions are satisfied.

(1) $\xi \in C\left(\Psi_{1}\right)$.

(2) For $1 \leq i+j+k \leq \alpha$, the partial derivatives $D^{(i, j, k)} \xi$ are continuous on the set $\{(s, t, u): 0<t<s<1, u \in \mathbb{R}\}$.

(3) For $1 \leq i+j+k \leq \alpha, s \in(0,1]$ and $u \in \mathbb{R}$,

$$
\left(D^{(i, j, k)} \xi\right)(s, 0+, u) \quad \text { and } \quad\left(D^{(i, j, k)} \xi\right)(s, s-, u) \text { exist. }
$$

(4) For $1 \leq i+j+k \leq \alpha, t \in[0,1)$ and $u \in \mathbb{R}$,

$$
\left(D^{(i, j, k)} \xi\right)(1-, t, u) \text { and }\left(D^{(i, j, k)} \xi\right)(t+, t, u) \text { exist. }
$$

The class of functions $C^{\alpha}\left(\Psi_{2}\right)$ is defined in a similar manner.

We assume that the kernel $\kappa$ of $K$ defined in (2.1) has the following properties.

$\left(\mathrm{H}_{1}\right)$ The partial derivative

$$
\frac{\partial^{4} \kappa}{\partial u^{4}}
$$

is continuous on $\Psi$.

$\left(\mathrm{H}_{2}\right)$ Let

$$
\ell(s, t, u):=\frac{\partial \kappa(s, t, u)}{\partial u}, \quad m(s, t, u):=\frac{\partial^{2} \kappa(s, t, u)}{\partial u^{2}} .
$$


There are functions $\ell_{i}, m_{i} \in C^{\alpha}\left(\Psi_{i}\right), i=1,2$, with

$$
\ell(s, t, u)= \begin{cases}\ell_{1}(s, t, u) & (s, t, u) \in \Psi_{1}, \\ \ell_{2}(s, t, u) & (s, t, u) \in \Psi_{2}\end{cases}
$$

and

$$
m(s, t, u)= \begin{cases}m_{1}(s, t, u) & (s, t, u) \in \Psi_{1}, \\ m_{2}(s, t, u) & (s, t, u) \in \Psi_{2} .\end{cases}
$$

$\left(\mathrm{H}_{3}\right) \ell, m \in C(\Psi)$.

Then it can be shown that:

$\left(\mathrm{H}_{4}\right)$ There are two functions $\kappa_{i} \in C^{\alpha}\left(\Psi_{i}\right), i=1,2$, such that

$$
\kappa(s, t, u)= \begin{cases}\kappa_{1}(s, t, u) & (s, t, u) \in \Psi_{1}, \\ \kappa_{2}(s, t, u) & (s, t, u) \in \Psi_{2} .\end{cases}
$$

Under the above assumptions, the operator $K$ is Fréchet differentiable, and its Fréchet derivative at $x \in L^{\infty}[0,1]$ is given by

$$
\begin{gathered}
\left(K^{\prime}(x) g\right)(s)=\int_{0}^{1} \frac{\partial \kappa(s, t, x(t))}{\partial u} g(t) d t, \\
s \in[0,1], \quad g \in L^{\infty}[0,1] .
\end{gathered}
$$

The operator $K^{\prime}$ is Lipschitz continuous in any bounded neighborhood $V$ of $\varphi$, that is, there exists a constant $\gamma$ such that

$$
\left\|K^{\prime}(x)-K^{\prime}(y)\right\| \leq \gamma\|x-y\|_{\infty}, \quad x, y \in V .
$$

Assume that, for $f \in X$,

$$
x-K(x)=f
$$

has a unique solution $\varphi$. We are interested in approximate solutions of the above equation.

From now on, we assume that $f \in C^{\alpha}[0,1]$. Then, by [ 4 , Corollary $3.2]$, it follows that

$$
\varphi \in C^{\alpha}[0,1] .
$$


We have:

$$
\begin{aligned}
\left(K^{\prime}(\varphi) g\right)(s) & =\int_{0}^{1} \frac{\partial \kappa(s, t, \varphi(t))}{\partial u} g(t) d t \\
& =\int_{0}^{1} \ell(s, t, \varphi(t)) g(t) d t, \quad s \in[0,1]
\end{aligned}
$$

Define

$$
\begin{aligned}
& \Omega_{1}=\{(s, t): 0 \leq t \leq s \leq 1\} \\
& \Omega_{2}=\{(s, t): 0 \leq s \leq t \leq 1\}
\end{aligned}
$$

and

$$
\ell_{*}(s, t):=\ell(s, t, \varphi(t))= \begin{cases}\ell_{1, *}(s, t)=\ell_{1}(s, t, \varphi(t)), & (s, t) \in \Omega_{1}, \\ \ell_{2, *}(s, t)=\ell_{2}(s, t, \varphi(t)), & (s, t) \in \Omega_{2} .\end{cases}
$$

By assumption $\left(\mathrm{H}_{3}\right)$,

$$
\ell_{*} \in C([0,1] \times[0,1]) .
$$

Since $\varphi \in C^{\alpha}[0,1]$, it follows that

$$
\ell_{1, *} \in C^{\alpha}\left(\Omega_{1}\right) \text { and } \ell_{2, *} \in C^{\alpha}\left(\Omega_{2}\right) .
$$

In the notation of Chatelin and Lebbar [5], a kernel satisfying (2.5) and (2.6) is said to be of the class $\mathcal{G}(\alpha, 0)$.

Note that the linear operator $K^{\prime}(\varphi): L^{\infty}[0,1] \rightarrow C[0,1]$ is compact.

For $x \in L^{\infty}[0,1]$, the second derivative $K^{\prime \prime}(x)$ is a bi-linear operator and is given by

$$
\begin{gathered}
\left(K^{\prime \prime}(x)\left(g_{1}, g_{2}\right)\right)(s)=\int_{0}^{1} \frac{\partial^{2} \kappa(s, t, x(t))}{\partial u^{2}} g_{1}(t) g_{2}(t) d t \\
g_{1}, g_{2} \in L^{\infty}[0,1] .
\end{gathered}
$$

Hence, for $s \in[0,1]$,

$$
\begin{aligned}
\left(K^{\prime \prime}(\varphi)\left(g_{1}, g_{2}\right)\right)(s) & =\int_{0}^{1} \frac{\partial^{2} \kappa(s, t, \varphi(t))}{\partial u^{2}} g_{1}(t) g_{2}(t) d t \\
& =\int_{0}^{1} m(s, t, \varphi(t)) g_{1}(t) g_{2}(t) d t
\end{aligned}
$$


Let

$$
m_{*}(s, t):=m(s, t, \varphi(t))= \begin{cases}m_{1, *}(s, t)=m_{1}(s, t, \varphi(t)), & (s, t) \in \Omega_{1}, \\ m_{2, *}(s, t)=m_{2}(s, t, \varphi(t)), & (s, t) \in \Omega_{2} .\end{cases}
$$

By assumption $\left(\mathrm{H}_{3}\right), m_{*} \in C([0,1] \times[0,1])$. Since $\varphi \in C^{\alpha}[0,1]$, it follows that $m_{1, *} \in C^{\alpha}\left(\Omega_{1}\right)$ and $m_{2, *} \in C^{\alpha}\left(\Omega_{2}\right)$. Thus, $m_{*} \in \mathcal{G}(\alpha, 0)$.

The third and the fourth derivatives of $K$ are given by

$$
\begin{aligned}
\left(K^{(3)}(x)\left(g_{1}, g_{2}, g_{3}\right)\right)(s) & =\int_{0}^{1} \frac{\partial^{3} \kappa(s, t, x(t))}{\partial u^{3}} g_{1}(t) g_{2}(t) g_{3}(t) d t, \\
s \in[0,1] &
\end{aligned}
$$

and

$$
\begin{aligned}
\left(K^{(4)}(x)\left(g_{1}, g_{2}, g_{3}, g_{4}\right)\right)(s)= & \int_{0}^{1} \frac{\partial^{4} \kappa(s, t, x(t))}{\partial u^{4}} g_{1}(t) g_{2}(t) g_{3}(t) g_{4}(t) d t \\
& s \in[0,1],
\end{aligned}
$$

where $x, g_{1}, g_{2}, g_{3}, g_{4} \in L^{\infty}[0,1]$.

2.2. Approximating space. For any integer $n$, let $\Delta^{(n)}: 0=t_{0}^{(n)}<$ $t_{1}^{(n)}<\cdots<t_{n}^{(n)}=1$ be a quasi-uniform partition of [0,1]. Define $h_{j}^{(n)}=t_{j}^{(n)}-t_{j-1}^{(n)}$,

$$
h^{(n)}=\max _{1 \leq j \leq n} h_{j}^{(n)}, \quad q^{(n)}=\max _{1 \leq i, j \leq n} \frac{h_{i}^{(n)}}{h_{j}^{(n)}} .
$$

Since the partition $\Delta^{(n)}$ is quasi-uniform, it follows that $\sup _{n} q^{(n)}<\infty$. For simplicity, we drop the index $n$ and write

$$
t_{j}=t_{j}^{(n)}, \Delta=\Delta^{(n)}, \Delta_{j}=\Delta_{j}^{(n)}=\left[t_{j-1}, t_{j}\right], h_{j}=t_{j}-t_{j-1}
$$

and

$$
h=\max _{1 \leq j \leq n} h_{j}
$$

Let $r \geq 0$ and $\mathcal{P}_{r, \Delta}$ denote the space of piecewise polynomials of degree $\leq r$ on each of the subintervals $\Delta_{j}, j=1,2, \ldots, n$. Then, $\mathcal{P}_{r, \Delta}$ is a subspace of $L^{\infty}[0,1]$. 
For $\nu \geq 0$, set

$$
C_{\Delta}^{\nu}=\left\{g \in L^{\infty}[0,1]:\left.g\right|_{\Delta_{j}} \in C^{\nu}\left(\Delta_{j}\right), j=1, \ldots, n\right\} .
$$

For $g \in C_{\Delta}^{\nu}$, we write $g_{j}=\left.g\right|_{\Delta_{j}}$ and, for $g \in C_{\Delta}=C_{\Delta}^{0}$, we introduce the following notation:

$$
\|g\|_{2, \Delta_{j}}:=\left\|g_{j}\right\|_{2}, \quad\|g\|_{\infty, \Delta_{j}}:=\left\|g_{j}\right\|_{\infty}, \quad\|g\|_{\infty}:=\max _{1 \leq j \leq n}\left\|g_{j}\right\|_{\infty} .
$$

Let $g \in C_{\Delta}$. Since

$$
\left(K^{\prime}(\varphi) g\right)(s)=\int_{0}^{s} \ell_{1, *}(s, t) g(t) d t+\int_{s}^{1} \ell_{2, *}(s, t) g(t) d t,
$$

we obtain

$$
\begin{aligned}
\left(K^{\prime}(\varphi) g\right)^{\prime}(s)= & \ell_{1, *}(s, s) g(s-)-\ell_{2, *}(s, s) g(s+) \\
& +\int_{0}^{s} \frac{\partial \ell_{1, *}(s, t)}{\partial s} g(t) d t+\int_{s}^{1} \frac{\partial \ell_{2, *}(s, t)}{\partial s} g(t) d t, \quad s \in[0,1] .
\end{aligned}
$$

If $s \notin \Delta$, then $g$ is continuous at $s$, that is, $g(s-)=g(s+)$. Since $\ell_{*} \in C([0,1] \times[0,1])$, we have $\ell_{1, *}(s, s)=\ell_{2, *}(s, s)$. Hence,

$$
\left(K^{\prime}(\varphi) g\right)^{\prime}(s)=\int_{0}^{s} \frac{\partial \ell_{1, *}(s, t)}{\partial s} g(t) d t+\int_{s}^{1} \frac{\partial \ell_{2, *}(s, t)}{\partial s} g(t) d t,
$$

and

$$
\begin{aligned}
\left(K^{\prime}(\varphi) g\right)^{\prime \prime}(s) & =\left(\frac{\partial \ell_{1, *}(s, s)}{\partial s}-\frac{\partial \ell_{2, *}(s, s)}{\partial s}\right) g(s) \\
& +\int_{0}^{s} \frac{\partial^{2} \ell_{1, *}(s, t)}{\partial s^{2}} g(t) d t+\int_{s}^{1} \frac{\partial^{2} \ell_{2, *}(s, t)}{\partial s^{2}} g(t) d t .
\end{aligned}
$$

For $s \in \Delta$, using limits, the values of $\left(K^{\prime}(\varphi) g\right)^{\prime \prime}(s+)$ and $\left(K^{\prime}(\varphi) g\right)^{\prime \prime}(s-)$ exist.

Thus, if $g \in C_{\Delta}$, then $K^{\prime}(\varphi) g \in C_{\Delta}^{2}$, and

$$
\left\|\left(K^{\prime}(\varphi) g\right)^{(j)}\right\|_{\infty} \leq C_{1}\|g\|_{\infty}, \quad j=0,1,2 .
$$

Since the kernel $m_{*}$ of $K^{\prime \prime}(\varphi)$ is also of the class $\mathcal{G}(\alpha, 0)$, it can be seen that, if $g_{1}, g_{2} \in C_{\Delta}$, then $K^{\prime \prime}(\varphi)\left(g_{1}, g_{2}\right) \in C_{\Delta}^{2}$ and

$$
\left\|\left(K^{\prime \prime}(\varphi)\left(g_{1}, g_{2}\right)\right)^{(j)}\right\|_{\infty} \leq C_{2}\left\|g_{1}\right\|_{\infty}\left\|g_{2}\right\|_{\infty}, \quad j=0,1,2 .
$$


Let $\ell_{*} \in \mathcal{G}(\alpha, 0)$ for $\alpha \geq 4$ and $g \in C_{\Delta}^{2}$. Then, for $s \notin \Delta$, we obtain

$$
\begin{aligned}
\left(K^{\prime}(\varphi) g\right)^{(3)}(s)= & 2\left(\frac{\partial^{2} \ell_{1, *}(s, s)}{\partial s^{2}}-\frac{\partial^{2} \ell_{2, *}(s, s)}{\partial s^{2}}\right) g(s) \\
& +\left(\frac{\partial \ell_{1, *}(s, s)}{\partial s}-\frac{\partial \ell_{2, *}(s, s)}{\partial s}\right) g^{\prime}(s) \\
& +\int_{0}^{s} \frac{\partial^{3} \ell_{1, *}(s, t)}{\partial s^{3}} g(t) d t+\int_{s}^{1} \frac{\partial^{3} \ell_{2, *}(s, t)}{\partial s^{3}} g(t) d t
\end{aligned}
$$

and

$$
\begin{aligned}
\left(K^{\prime}(\varphi) g\right)^{(4)}(s)= & 3\left(\frac{\partial^{3} \ell_{1, *}(s, s)}{\partial s^{3}}-\frac{\partial^{3} \ell_{2, *}(s, s)}{\partial s^{3}}\right) g(s) \\
& +3\left(\frac{\partial^{2} \ell_{1, *}(s, s)}{\partial s^{2}}-\frac{\partial^{2} \ell_{2, *}(s, s)}{\partial s^{2}}\right) g^{\prime}(s) \\
& +\left(\frac{\partial \ell_{1, *}(s, s)}{\partial s}-\frac{\partial \ell_{2, *}(s, s)}{\partial s}\right) g^{\prime \prime}(s) \\
& +\int_{0}^{s} \frac{\partial^{4} \ell_{1, *}(s, t)}{\partial s^{4}} g(t) d t+\int_{s}^{1} \frac{\partial^{4} \ell_{2, *}(s, t)}{\partial s^{4}} g(t) d t .
\end{aligned}
$$

As a consequence, if $\ell_{*} \in \mathcal{G}(\alpha, 0)$ for $\alpha \geq 4$ and $g \in C_{\Delta}^{2}$, then

$$
\begin{aligned}
& \left\|\left(K^{\prime}(\varphi) g\right)^{(3)}\right\|_{\infty} \leq C_{3}\left(\|g\|_{\infty}+\left\|g^{\prime}\right\|_{\infty}\right), \\
& \left\|\left(K^{\prime}(\varphi) g\right)^{(4)}\right\|_{\infty} \leq C_{3}\left(\|g\|_{\infty}+\left\|g^{\prime}\right\|_{\infty}+\left\|g^{\prime \prime}\right\|_{\infty}\right) .
\end{aligned}
$$

2.3. Interpolatory projection at Gauss points. For $j=1,2, \ldots, n$, let $\tau_{1}^{j}<\tau_{2}^{j}<\cdots<\tau_{r+1}^{j}$ be the Gauss-Legendre points in $\left[t_{j-1}, t_{j}\right]$. Let $A=\left\{\tau_{p}^{j}, p=1,2, \ldots, r+1, j=1,2, \ldots, n\right\}$ be the set of the collocation points. The interpolatory projection $Q_{n}: C_{\Delta} \rightarrow \mathcal{P}_{r, \Delta}$ is defined as follows:

$$
\begin{gathered}
Q_{n} g \in \mathcal{P}_{r, \Delta}, \quad\left(Q_{n} g\right)\left(\tau_{p}^{j}\right)=g\left(\tau_{p}^{j}\right), \\
1 \leq p \leq r+1, \quad 1 \leq j \leq n .
\end{gathered}
$$

Then

$$
\sup _{n}\left\|\left.Q_{n}\right|_{C_{\Delta}}\right\|<\infty
$$

Also, for $g \in C[0,1]$,

$$
\left\|\left(I-Q_{n}\right) g\right\|_{\infty} \longrightarrow 0 \quad \text { as } n \rightarrow \infty .
$$


Let $P_{n}$ be the restriction to $L^{\infty}[0,1]$ of the orthogonal projection from $L^{2}[0,1]$ onto $\mathcal{P}_{r, \Delta}$. We define

$$
\beta=\min \{\alpha, r+1\}
$$

The following result is quoted from [4, Corollary 4.3].

Lemma 2.1. Let $\left\{\pi_{n}\right\}$ be a sequence of projections from $C_{\Delta}$ onto $\mathcal{P}_{r, \Delta}$ such that

$$
\sup _{n}\left\|\pi_{n}\right\|_{\infty}<\infty
$$

Then there is a constant $C_{4}$ such that, for any $g \in C_{\Delta}^{\alpha}$,

$$
\left\|\left(I-\pi_{n}\right) g\right\|_{\infty} \leq C_{4}\left\|g^{(\beta)}\right\|_{\infty} h^{\beta} .
$$

Thus,

$$
\left\|\left(I-Q_{n}\right) g\right\|_{\infty} \leq C_{4}\left\|g^{(p)}\right\|_{\infty} h^{p}, \quad 1 \leq p \leq \beta .
$$

Let $Q_{n, j} y=\left.\left(Q_{n} y\right)\right|_{\Delta_{j}}, P_{n, j} y=\left.\left(P_{n} y\right)\right|_{\Delta_{j}}, y \in C_{\Delta}$. Then

$$
\sup _{n, j}\left\|P_{n, j}\right\|_{\infty}<\infty .
$$

The following result is deduced from Lemma 2.1.

Lemma 2.2. Let $g \in C_{\Delta}^{\alpha}$ and $g_{j}=\left.g\right|_{\Delta_{j}}$. Then there is a constant $C_{5}$ such that

$$
\left\|\left(I-P_{n, j}\right) g_{j}\right\|_{\infty, \Delta_{j}} \leq C_{5}\left\|g_{j}^{(p)}\right\|_{\infty, \Delta_{j}} h_{j}^{p}, \quad 1 \leq j \leq n, 1 \leq p \leq \beta .
$$

For $g \in C\left(\Delta_{j}\right)$ and for $s \in \Delta_{j}$, let $\delta_{j}^{r+1} g(s)=\left[\tau_{1}^{j}, \ldots, \tau_{r+1}^{j}, s\right] g$ denote the divided difference of $g$ at $\left\{\tau_{1}^{j}, \ldots, \tau_{r+1}^{j}, s\right\}$.

We state the following important result from [5] for future reference. 
Lemma 2.3. (Chatelin-Lebbar [5]). For $f, g \in C\left(\Delta_{j}\right)$,

$$
\left\langle\left(I-Q_{n, j}\right) g, \bar{f}\right\rangle_{j}=\left\langle\left(I-P_{n, j}\right) f \delta_{j}^{r+1} g, v_{j}\right\rangle_{j},
$$

where

$$
v_{j}(t)=\prod_{p=1}^{r+1}\left(t-\tau_{p}^{j}\right)
$$

and $\langle\cdot, \cdot\rangle_{j}$ denotes the inner product of $L^{2}\left(\Delta_{j}\right)$.

2.4. Projection methods. Let $K$ be a Urysohn integral operator with a kernel $\kappa$ satisfying assumptions $\left(\mathrm{H}_{1}\right),\left(\mathrm{H}_{2}\right)$ and $\left(\mathrm{H}_{3}\right)$. Let $Q_{n}$ be the interpolatory projection at $r+1$ Gauss points defined by (2.15).

In [4], the collocation and the iterated collocation methods are investigated and, under slightly weaker assumptions on the kernel $\kappa$ as compared to our assumptions, the following orders of convergence are proved:

$$
\left\|\varphi_{n}^{C}-\varphi\right\|_{\infty}=O\left(h^{\beta}\right),
$$

and, if $\alpha \geq r+1$, then

$$
\left\|\varphi_{n}^{S}-\varphi\right\|_{\infty}=O\left(h^{\min \{\alpha, 2 r+2, r+3\}}\right) .
$$

Consider the following modified projection method from [7]:

$$
\varphi_{n}^{M}-K_{n}^{M}\left(\varphi_{n}^{M}\right)=f,
$$

where

$$
K_{n}^{M}(x)=Q_{n} K(x)+K\left(Q_{n} x\right)-Q_{n} K\left(Q_{n} x\right), \quad x \in X .
$$

The iterated modified projection solution is defined as

$$
\widetilde{\varphi}_{n}^{M}=K\left(\varphi_{n}^{M}\right)+f .
$$

As it is explained in [8, Section 4], the size of the system that needs to be solved in the modified projection method remains the same as in the collocation method, even though one needs to generate additional matrices and the right hand of the system has an extra term. The iterated modified projection solution is obtained by performing one step of iteration, and thus there is no additional system to be solved. 
For $\delta>0$, let $\mathcal{B}(\varphi, \delta)=\left\{\psi \in X:\|\varphi-\psi\|_{\infty}<\delta\right\}$. In order to prove the existence of $\varphi_{n}^{M}$ in a neighborhood of $\varphi$, we quote the following result from Grammont [6].

Theorem 2.4. Let $K$ be a completely continuous operator defined on the closure $\bar{D}$ of an open subset $D$ of a Banach space $X$. Let $Y$ be a closed subspace of $X$ such that $K(x) \in Y$ for all $x \in \bar{D}$. Assume that $x=K(x)$ has a solution $x^{*}$ in $D$. Further assume that $K$ is Fréchet differentiable in $D$, the Fréchet derivative $K^{\prime}$ is Lipschitz continuous in $D$ and that 1 is not an eigenvalue of $K^{\prime}\left(x^{*}\right)$. Let $X_{n}$ be a sequence of finite-dimensional subspaces of $X$ and $Q_{n}: X \rightarrow X_{n}$ be a sequence of projections such that $\left\|Q_{n} y-y\right\| \rightarrow 0$ as $n \rightarrow \infty$ for all $y \in Y$. Then there exists $\delta_{0}>0$ such that $K_{n}^{M}$ has a unique fixed point $x_{n}^{M}$ in $\mathcal{B}\left(x^{*}, \delta_{0}\right)$ and that

$$
\frac{2}{3} \alpha_{n} \leq\left\|x_{n}^{M}-x^{*}\right\|_{\infty} \leq 2 \alpha_{n},
$$

where $\alpha_{n}=\left\|\left[I-\left(K_{n}^{M}\right)^{\prime}\left(x^{*}\right)\right]^{-1}\left[K\left(x^{*}\right)-K_{n}^{M}\left(x^{*}\right)\right]\right\|$ is a sequence converging to zero.

The proof of the above theorem can easily be adapted to prove the following result.

Theorem 2.5. Let $K$ be a Urysohn integral operator with a continuous kernel $\kappa$ satisfying assumptions $\left(H_{1}\right),\left(H_{2}\right)$ and $\left(H_{3}\right)$. Let $\varphi$ be the unique solution of (2.3), and assume that 1 is not an eigenvalue of $K^{\prime}(\varphi)$. Let $Q_{n}$ be the interpolatory projection at $r+1$ Gauss points defined by (2.15). Then there exists a neighbourhood $\mathcal{B}\left(\varphi, \delta_{0}\right)$ of $\varphi$ which contains, for all $n$ large enough, a unique solution $\varphi_{n}^{M}$ of (2.24). Further,

$$
\frac{2}{3} \alpha_{n} \leq\left\|\varphi_{n}^{M}-\varphi\right\|_{\infty} \leq 2 \alpha_{n},
$$

where $\alpha_{n}=\left\|\left[I-\left(K_{n}^{M}\right)^{\prime}(\varphi)\right]^{-1}\left[K(\varphi)-K_{n}^{M}(\varphi)\right]\right\|$ is a sequence converging to zero.

The following result is needed in subsection 4.1 for obtaining the order of convergence of the modified projection solution. 
Proposition 2.6. Let $K$ be a Urysohn integral operator with a continuous kernel $\kappa$ satisfying assumptions $\left(H_{1}\right),\left(H_{2}\right)$ and $\left(H_{3}\right)$. Let $\varphi$ be the unique solution of (2.3), and assume that 1 is not an eigenvalue of $K^{\prime}(\varphi)$. Let $Q_{n}$ be the interpolatory projection at $r+1$ Gauss points defined by (2.15). Then there exists a positive integer $n_{1}$ such that, for $n \geq n_{1}$, the operator $I-\left(K_{n}^{M}\right)^{\prime}(\varphi)$ is invertible and $\left\|\left(I-\left(K_{n}^{M}\right)^{\prime}(\varphi)\right)^{-1}\right\| \leq 2\left\|\left(I-K^{\prime}(\varphi)\right)^{-1}\right\|$.

Proof. Note that $\left(K_{n}^{M}\right)^{\prime}(\varphi)=Q_{n} K^{\prime}(\varphi)+\left(I-Q_{n}\right) K^{\prime}\left(Q_{n} \varphi\right) Q_{n}$. Hence,

$$
\begin{aligned}
& K^{\prime}(\varphi)-\left(K_{n}^{M}\right)^{\prime}(\varphi) \\
& \quad=\left(I-Q_{n}\right) K^{\prime}(\varphi)\left(I-Q_{n}\right)+\left(I-Q_{n}\right)\left(K^{\prime}(\varphi)-K^{\prime}\left(Q_{n} \varphi\right)\right) Q_{n} .
\end{aligned}
$$

Since $K^{\prime}(\varphi): L^{\infty}[0,1] \rightarrow C[0,1]$ is a compact linear operator and since, by $(2.17), Q_{n}$ converges to the identity operator pointwise on $C[0,1]$, it follows that

$$
\left\|\left(I-Q_{n}\right) K^{\prime}(\varphi)\right\| \longrightarrow 0 \quad \text { as } n \rightarrow \infty .
$$

Choose a positive integer $n_{0}$ such that $Q_{n} \varphi \in \mathcal{B}\left(\varphi, \delta_{0}\right)$ for $n \geq n_{0}$. Then, by (2.2),

$$
\left\|K^{\prime}(\varphi)-K^{\prime}\left(Q_{n} \varphi\right)\right\| \leq \gamma\left\|\varphi-Q_{n} \varphi\right\|_{\infty} \longrightarrow 0 \quad \text { as } n \rightarrow \infty .
$$

Since $\sup _{n}\left\|\left.Q_{n}\right|_{C_{\Delta}}\right\|<\infty$, it follows that $\left\|K^{\prime}(\varphi)-\left(K_{n}^{M}\right)^{\prime}(\varphi)\right\| \rightarrow 0$ as $n \rightarrow \infty$. Choose $n_{1} \geq n_{0}$ such that $\left\|K^{\prime}(\varphi)-\left(K_{n}^{M}\right)^{\prime}(\varphi)\right\| \|(I-$ $\left.K^{\prime}(\varphi)\right)^{-1} \| \leq 1 / 2$ for $n \geq n_{1}$. Since

$I-\left(K_{n}^{M}\right)^{\prime}(\varphi)=\left[I-\left\{\left(K_{n}^{M}\right)^{\prime}(\varphi)-K^{\prime}(\varphi)\right\}\left(I-K^{\prime}(\varphi)\right)^{-1}\right]\left(I-K^{\prime}(\varphi)\right)$,

it follows that $\left\|\left(I-\left(K_{n}^{M}\right)^{\prime}(\varphi)\right)^{-1}\right\| \leq 2\left\|\left(I-K^{\prime}(\varphi)\right)^{-1}\right\|$ for $n \geq n_{1}$. This completes the proof.

For future reference, let

$$
\begin{aligned}
& \sup _{\substack{s, t \in[0,1] \\
|u| \leq\|\varphi\|_{\infty}+\delta_{0}}}\left|\frac{\partial^{2} \kappa}{\partial u^{2}}(s, t, u)\right|=M_{1}, \\
& \sup _{\substack{s, t \in[0,1] \\
|u| \leq\|\varphi\|_{\infty}+\delta_{0}}}\left|\frac{\partial^{3} \kappa}{\partial u^{3}}(s, t, u)\right|=M_{2}
\end{aligned}
$$


and

$$
\sup _{\substack{s, t \in[0,1] \\|u| \leq\|\varphi\|_{\infty}+\delta_{0}}}\left|\frac{\partial^{4} \kappa}{\partial u^{4}}(s, t, u)\right|=M_{3} .
$$

3. Error estimates. In this section, we prove error estimates which are needed to obtain the orders of convergence of the modified projection and the iterated modified projection solutions. In subsection 3.1, we obtain error bounds for the divided difference of $K^{\prime}(\varphi) g_{1}$ for $g_{1} \in C[0,1]$ and for similar quantities associated with the second and the third Fréchet derivatives of $K$ at $\varphi$. Based on these bounds, we prove a crucial lemma in subsection 3.2 which is used in proving many results which follow. In subsection 3.3, we show that, for the case piecewise constant polynomials, that is, when $r=0$, some orders of convergence obtained in subsection 3.2 can be improved.

3.1. Divided difference. Let $g \in C[0,1], \zeta_{1}, \ldots, \zeta_{r+1}$ be distinct points in $[0,1]$ and $s \in[0,1]$. The divided difference of $g$ at $\zeta_{1}, \ldots, \zeta_{r+1}$ and $s$ is denoted by $\left[\zeta_{1}, \ldots, \zeta_{r+1}, s\right] g$.

We first prove two important results. The crucial idea is that

$$
\left[\zeta_{1}, \ldots, \zeta_{r+1}, s\right]\left(K^{\prime}(\varphi) g\right)=\int_{0}^{1}\left[\zeta_{1}, \ldots, \zeta_{r+1}, s\right] \ell_{*}(\cdot, t) g(t) d t
$$

where $\left[\zeta_{1}, \ldots, \zeta_{r+1}, s\right] \ell_{*}(\cdot, t)$ denotes the divided difference of $\ell_{*}$ with respect to the first variable.

Lemma 3.1. Let $r \geq 0, \zeta_{1}, \ldots, \zeta_{r+1}$ be distinct points in $(0,1)$ and $g_{1}, g_{2}, g_{3} \in C[0,1]$. Then

$$
\begin{gathered}
\sup _{s \in[0,1]}\left|\left[\zeta_{1}, \ldots, \zeta_{r+1}, s\right]\left(K^{\prime}(\varphi) g_{1}\right)\right| \leq C_{6}\left\|g_{1}\right\|_{\infty} \\
\sup _{s \in[0,1]}\left|\left[\zeta_{1}, \ldots, \zeta_{r+1}, s\right]\left(K^{\prime \prime}(\varphi)\left(g_{1}, g_{2}\right)\right)\right| \leq C_{7}\left\|g_{1}\right\|_{\infty}\left\|g_{2}\right\|_{\infty}
\end{gathered}
$$

$$
\sup _{s \in[0,1]}\left|\left[\zeta_{1}, \ldots, \zeta_{r+1}, s\right]\left(K^{(3)}(\varphi)\left(g_{1}, g_{2}, g_{3}\right)\right)\right| \leq C_{8}\left\|g_{1}\right\|_{\infty}\left\|g_{2}\right\|_{\infty}\left\|g_{3}\right\|_{\infty}
$$

where $C_{6}, C_{7}$ and $C_{8}$ are constants. 
Proof. The proof is by induction. If $s \neq \zeta_{1}$, then it can be easily verified that

$$
\left[\zeta_{1}, s\right]\left(K^{\prime}(\varphi) g_{1}\right)=\int_{0}^{1}\left[\zeta_{1}, s\right] \ell_{*}(\cdot, t) g_{1}(t) d t, \quad s \in[0,1]
$$

where

$$
\left[\zeta_{1}, s\right] \ell_{*}(\cdot, t)=\frac{\ell_{*}(s, t)-\ell_{*}\left(\zeta_{1}, t\right)}{s-\zeta_{1}}
$$

Let

$$
\begin{aligned}
& M_{4}=\sup \left\{\left|D^{(1,0)} \ell_{1, *}(s, t)\right|: 0 \leq t \leq s \leq 1\right\}, \\
& M_{5}=\sup \left\{\left|D^{(1,0)} \ell_{2, *}(s, t)\right|: 0 \leq s \leq t \leq 1\right\} .
\end{aligned}
$$

Fix $s \in[0,1]$.

Case $1.0 \leq s<\zeta_{1}$. Note that

$$
\left[\zeta_{1}, s\right] \ell_{*}(\cdot, t)=\frac{\ell_{*}(s, t)-\ell_{*}\left(\zeta_{1}, t\right)}{s-\zeta_{1}}= \begin{cases}\frac{\ell_{1, *}(s, t)-\ell_{1, *}\left(\zeta_{1}, t\right)}{s-\zeta_{1}} & 0 \leq t \leq s \\ \frac{\ell_{2, *}(s, t)-\ell_{1, *}\left(\zeta_{1}, t\right)}{s-\zeta_{1}} & s<t<\zeta_{1} \\ \frac{\ell_{2, *}(s, t)-\ell_{2, *}\left(\zeta_{1}, t\right)}{s-\zeta_{1}} & \zeta_{1} \leq t \leq 1\end{cases}
$$

Thus, for a fixed $s, 0 \leq s<\zeta_{1}$, the function $\left[\zeta_{1}, s\right] \ell_{*}(\cdot, t)$ is continuous on $[0,1]$.

By the mean value theorem,

$$
\frac{\ell_{1, *}(s, t)-\ell_{1, *}\left(\zeta_{1}, t\right)}{s-\zeta_{1}}=D^{(1,0)} \ell_{1, *}\left(\eta_{1}, t\right)
$$

and

$$
\frac{\ell_{2, *}(s, t)-\ell_{2, *}\left(\zeta_{1}, t\right)}{s-\zeta_{1}}=D^{(1,0)} \ell_{2, *}\left(\xi_{1}, t\right),
$$

where $\eta_{1}, \xi_{1} \in\left(s, \zeta_{1}\right)$. Hence,

$$
\left|\left[\zeta_{1}, s\right] \ell_{*}(\cdot, t)\right| \leq M_{4} \quad \text { if } 0 \leq t \leq s
$$

and

$$
\left|\left[\zeta_{1}, s\right] \ell_{*}(\cdot, t)\right| \leq M_{5} \quad \text { if } \zeta_{1} \leq t \leq 1
$$


On the other hand, for $s<t<\zeta_{1}$,

$$
\begin{aligned}
\frac{\ell_{2, *}(s, t)-\ell_{1, *}\left(\zeta_{1}, t\right)}{s-\zeta_{1}}= & \frac{\ell_{2, *}(s, t)-\ell_{2, *}(t, t)+\ell_{1, *}(t, t)-\ell_{1, *}\left(\zeta_{1}, t\right)}{s-\zeta_{1}} \\
= & \frac{D^{(1,0)} \ell_{2, *}\left(\xi_{2}, t\right)(s-t)}{s-\zeta_{1}} \\
& +\frac{D^{(1,0)} \ell_{1, *}\left(\eta_{2}, t\right)\left(t-\zeta_{1}\right)}{s-\zeta_{1}}
\end{aligned}
$$

where $\xi_{2} \in(s, t)$ and $\eta_{2} \in\left(t, \zeta_{1}\right)$. Hence, if $s<t<\zeta_{1}$, then

$$
\left|\frac{\ell_{2, *}(s, t)-\ell_{1, *}\left(\zeta_{1}, t\right)}{s-\zeta_{1}}\right| \leq M_{4}+M_{5}
$$

Thus, $\sup \left\{\left|\left[\zeta_{1}, s\right] \ell_{*}(\cdot, t)\right|: t \in[0,1]\right\} \leq M_{4}+M_{5}$, and hence,

$$
\begin{aligned}
\left|\left[\zeta_{1}, s\right]\left(K^{\prime}(\varphi) g_{1}\right)\right| & =\left|\int_{0}^{1}\left[\zeta_{1}, s\right] \ell_{*}(\cdot, t) g_{1}(t) d t\right| \\
& \leq\left(M_{4}+M_{5}\right)\left\|g_{1}\right\|_{\infty} .
\end{aligned}
$$

Case 2. $s=\zeta_{1}$. In this case,

$$
\left[\zeta_{1}, \zeta_{1}\right] \ell_{*}(\cdot, t)=\frac{\partial \ell_{*}\left(\zeta_{1}, t\right)}{\partial s}= \begin{cases}\frac{\partial \ell_{1, *}\left(\zeta_{1}, t\right)}{\partial s} & 0 \leq t<\zeta_{1}<1 \\ \frac{\partial \ell_{2, *}\left(\zeta_{1}, t\right)}{\partial s} & 0<\zeta_{1}<t \leq 1\end{cases}
$$

The above function is possibly discontinuous at $t=\zeta_{1}$. We obtain

$$
\begin{aligned}
\left|\left[\zeta_{1}, \zeta_{1}\right]\left(K^{\prime}(\varphi) g_{1}\right)\right| & =\left|\int_{0}^{\zeta_{1}} \frac{\partial \ell_{1, *}\left(\zeta_{1}, t\right)}{\partial s} g_{1}(t) d t+\int_{\zeta_{1}}^{1} \frac{\partial \ell_{2, *}\left(\zeta_{1}, t\right)}{\partial s} g_{1}(t) d t\right| \\
& \left.\leq(3.6) \quad M_{4}+M_{5}\right)\left\|g_{1}\right\|_{\infty} .
\end{aligned}
$$

Case 3. $\zeta_{1}<s \leq 1$. As in Case 1, it can be shown that

$$
\left|\left[\zeta_{1}, s\right]\left(K^{\prime}(\varphi) g_{1}\right)\right| \leq\left(M_{4}+M_{5}\right)\left\|g_{1}\right\|_{\infty} .
$$

From (3.5)-(3.7), we conclude that $\sup _{s \in[0,1]}\left|\left[\zeta_{1}, s\right]\left(K^{\prime}(\varphi) g_{1}\right)\right| \leq\left(M_{4}+\right.$ $\left.M_{5}\right)\left\|g_{1}\right\|_{\infty}$, which proves the estimate (3.1) for the case $r=0$.

Assume that, for any distinct $\zeta_{1}, \ldots, \zeta_{j}, j \leq r$, and for $s \in[0,1]$,

$$
\left|\left[\zeta_{1}, \ldots, \zeta_{j}, s\right]\left(K^{\prime}(\varphi) g_{1}\right)\right| \leq C_{6}\left\|g_{1}\right\|_{\infty} .
$$

Then, since 


$$
\begin{aligned}
& {\left[\zeta_{1}, \ldots, \zeta_{j}, \zeta_{j+1}, s\right]\left(K^{\prime}(\varphi) g_{1}\right)} \\
& =\frac{\left[\zeta_{2}, \ldots, \zeta_{j}, \zeta_{j+1}, s\right]\left(K^{\prime}(\varphi) g_{1}\right)-\left[\zeta_{1}, \ldots, \zeta_{j}, s\right]\left(K^{\prime}(\varphi) g_{1}\right)}{\zeta_{j+1}-\zeta_{1}},
\end{aligned}
$$

it follows that

$$
\left|\left[\zeta_{1}, \ldots, \zeta_{j}, \zeta_{j+1}, s\right]\left(K^{\prime}(\varphi) g_{1}\right)\right| \leq \frac{2 C_{6}}{\left|\zeta_{j+1}-\zeta_{1}\right|}\left\|g_{1}\right\|_{\infty} .
$$

Since $s \in[0,1]$ is arbitrary, the proof of $(3.1)$ is complete. The proofs of (3.2) and (3.3) are similar.

Lemma 3.2. Let $r \geq 0$ and $\zeta_{1}, \ldots, \zeta_{r+1}$ be distinct points in $(0,1)$ and $g \in C[0,1]$. Then

$$
\sup _{s \in[0,1]}\left|\left[\zeta_{1}, \ldots, \zeta_{r+1}, s, s\right]\left(K^{\prime}(\varphi) g\right)\right| \leq C_{9}\|g\|_{\infty},
$$

where $C_{9}$ is a constant.

Proof. The proof is by induction. If $s \neq \zeta_{1}$, then

$$
\left[\zeta_{1}, s, s\right]\left(K^{\prime}(\varphi) g\right)=\int_{0}^{1}\left[\zeta_{1}, s, s\right] \ell_{*}(\cdot, t) g(t) d t, \quad s \in[0,1] .
$$

Let

$$
\begin{aligned}
& M_{6}=\sup \left\{\left|D^{(2,0)} \ell_{1, *}(s, t)\right|: 0 \leq t \leq s \leq 1\right\}, \\
& M_{7}=\sup \left\{\left|D^{(2,0)} \ell_{2, *}(s, t)\right|: 0 \leq s \leq t \leq 1\right\} .
\end{aligned}
$$

Fix $s \in[0,1]$.

Case 1. $0 \leq s<\zeta_{1}$. Note that

$$
\begin{aligned}
{\left[\zeta_{1}, s, s\right] \ell_{*}(\cdot, t)=} & \frac{[s, s] \ell_{*}(\cdot, t)-\left[\zeta_{1}, s\right] \ell_{*}(\cdot, t)}{s-\zeta_{1}} \\
= & \begin{cases}\frac{\partial \ell_{1, *}(s, t) / \partial s-\left(\ell_{1, *}(s, t)-\ell_{1, *}\left(\zeta_{1}, t\right)\right) /\left(s-\zeta_{1}\right)}{s-\zeta_{1}} & 0 \leq t \leq s, \\
\frac{\partial \ell_{2, *}(s, t) / \partial s-\left(\ell_{2, *}(s, t)-\ell_{1, *}\left(\zeta_{1}, t\right)\right) /\left(s-\zeta_{1}\right)}{s-\zeta_{1}} & s<t<\zeta_{1}, \\
\frac{\partial \ell_{2, *}(s, t) / \partial s-\left(\ell_{2, *}(s, t)-\ell_{2, *}\left(\zeta_{1}, t\right)\right) /\left(s-\zeta_{1}\right)}{s-\zeta_{1}} & \zeta_{1} \leq t \leq 1 .\end{cases}
\end{aligned}
$$

The above function is possibly discontinuous at $t=s$. 
For $0 \leq t \leq s$,

$$
\begin{aligned}
\frac{\partial \ell_{1, *}(s, t) / \partial s-\ell_{1, *}(s, t)-\ell_{1, *}\left(\zeta_{1}, t\right) s-\zeta_{1}}{s-\zeta_{1}} \\
=\frac{\partial \ell_{1, *}(s, t) / \partial s-\partial \ell_{1, *}\left(\eta_{3}, t\right) \partial s}{s-\zeta_{1}} \\
=\frac{\partial^{2} \ell_{1, *}\left(\eta_{4}, t\right) / \partial s^{2}\left(s-\eta_{3}\right)}{s-\zeta_{1}},
\end{aligned}
$$

where $\eta_{3} \in\left(s, \zeta_{1}\right)$ and $\eta_{4} \in\left(s, \eta_{3}\right) \subset\left(s, \zeta_{1}\right)$. Hence,

$$
\left|\left[\zeta_{1}, s, s\right] \ell_{*}(\cdot, t)\right| \leq M_{6} \text { if } 0 \leq t \leq s .
$$

In a similar manner, it follows that

$$
\left|\left[\zeta_{1}, s, s\right] \ell_{*}(\cdot, t)\right| \leq M_{7} \quad \text { if } \zeta_{1} \leq t \leq 1 .
$$

For $s<t<\zeta_{1}$, using (3.4), we obtain

$$
\left|\frac{\partial \ell_{2, *}(s, t)}{\partial s}-\frac{\ell_{2, *}(s, t)-\ell_{1, *}\left(\zeta_{1}, t\right)}{s-\zeta_{1}}\right| \leq M_{4}+2 M_{5} .
$$

Hence,

$$
\int_{s}^{\zeta_{1}}\left|\frac{\partial \ell_{2, *}(s, t) / \partial s-\ell_{2, *}(s, t)-\ell_{1, *}\left(\zeta_{1}, t\right) /\left(s-\zeta_{1}\right)}{s-\zeta_{1}}\right| d t \leq M_{4}+2 M_{5} .
$$

From (3.9)-(3.11), it follows that

$$
\begin{aligned}
\left|\left[\zeta_{1}, s, s\right]\left(K^{\prime}(\varphi) g\right)\right| & =\left|\int_{0}^{1}\left[\zeta_{1}, s, s\right] \ell_{*}(\cdot, t) g(t) d t\right| \\
& \leq\left(M_{4}+2 M_{5}+M_{6}+M_{7}\right)\|g\|_{\infty} .
\end{aligned}
$$

Case 2. $s=\zeta_{1}$. In this case, since $g \in C[0,1]$, using (2.11), we obtain

$$
\begin{aligned}
{\left[\zeta_{1}, \zeta_{1}, \zeta_{1}\right]\left(K^{\prime}(\varphi) g\right) } & =\frac{1}{2}\left(K^{\prime}(\varphi) g\right)^{\prime \prime}\left(\zeta_{1}\right) \\
& =\frac{1}{2}\left(\frac{\partial \ell_{1, *}\left(\zeta_{1}, \zeta_{1}\right)}{\partial s}-\frac{\partial \ell_{2, *}}{\partial s}\left(\zeta_{1}, \zeta_{1}\right)\right) g\left(\zeta_{1}\right)
\end{aligned}
$$




$$
+\frac{1}{2}\left[\int_{0}^{\zeta_{1}} \frac{\partial^{2} \ell_{1, *}\left(\zeta_{1}, t\right)}{\partial s^{2}} g(t) d t \quad+\int_{\zeta_{1}}^{1} \frac{\partial^{2} \ell_{2, *}\left(\zeta_{1}, t\right)}{\partial s^{2}} g(t) d t\right] .
$$

Hence,

$$
\left|\left[\zeta_{1}, \zeta_{1}, \zeta_{1}\right]\left(K^{\prime}(\varphi) g\right)\right| \leq \frac{M_{4}+M_{5}+M_{6}+M_{7}}{2}\|g\|_{\infty} .
$$

Case 3. $\zeta_{1}<s \leq 1$. As in Case 1, it can be seen that

$$
\left|\left[\zeta_{1}, s, s\right]\left(K^{\prime}(\varphi) g\right)\right| \leq\left(2 M_{4}+M_{5}+M_{6}+M_{7}\right)\|g\|_{\infty} .
$$

From (3.12)-(3.14), it follows that

$$
\sup _{s \in[0,1]}\left|\left[\zeta_{1}, s, s\right]\left(K^{\prime}(\varphi) g\right)\right| \leq\left(2 M_{4}+2 M_{5}+M_{6}+M_{7}\right)\|g\|_{\infty},
$$

which proves the required estimate for the case $r=0$.

Assume that, for any distinct $\zeta_{1}, \ldots, \zeta_{j}, j \leq r$, and for $s \in[0,1]$,

$$
\left|\left[\zeta_{1}, \ldots, \zeta_{j}, s, s\right]\left(K^{\prime}(\varphi) g\right)\right| \leq C_{9}\|g\|_{\infty} .
$$

Then, since

$$
\begin{aligned}
& {\left[\zeta_{1}, \ldots, \zeta_{j}, \zeta_{j+1}, s, s\right]\left(K^{\prime}(\varphi) g\right)} \\
& \quad=\frac{\left[\zeta_{2}, \ldots, \zeta_{j}, \zeta_{j+1}, s, s\right]\left(K^{\prime}(\varphi) g\right)-\left[\zeta_{1}, \ldots, \zeta_{j}, s, s\right]\left(K^{\prime}(\varphi) g\right)}{\zeta_{j+1}-\zeta_{1}},
\end{aligned}
$$

it follows that

$$
\left|\left[\zeta_{1}, \ldots, \zeta_{j}, \zeta_{j+1}, s, s\right]\left(K^{\prime}(\varphi) g\right)\right| \leq \frac{2 C_{9}}{\left|\zeta_{j+1}-\zeta_{1}\right|}\|g\|_{\infty} .
$$

This completes the proof.

3.2. Interpolation at $r+1$ Gauss points. We first prove a result which will be used in obtaining the order of convergence of the modified projection solution in subsection 4.1.

Proposition 3.3. Let $\alpha \geq 2$ and $g \in C_{\Delta}^{\alpha}$. Then

$$
\left\|\left(I-Q_{n}\right) K^{\prime}(\varphi)\left(I-Q_{n}\right) g\right\|_{\infty}=O\left(h^{\beta+2}\right) .
$$

Proof. If $r=0$, then $\beta=\min \{\alpha, r+1\}=1$ and, by (2.19),

$$
\left\|\left(I-Q_{n}\right) K^{\prime}(\varphi)\left(I-Q_{n}\right) g\right\|_{\infty} \leq C_{4}\left\|\left(K^{\prime}(\varphi)\left(I-Q_{n}\right) g\right)^{\prime}\right\|_{\infty} h .
$$


Since the kernel $\ell_{*}$ of the linear integral operator $K^{\prime}(\varphi)$ is of the class $\mathcal{G}(\alpha, 0)$, by [5, Theorem 15],

$$
\left\|\left(K^{\prime}(\varphi)\left(I-Q_{n}\right) g\right)^{\prime}\right\|_{\infty}=O\left(h^{2}\right) .
$$

Combining the above two estimates, we obtain the required estimate for the case $r=0$.

If $r \geq 1$, then $\beta \geq 2$. Recall from (2.12) that, if $x \in C_{\Delta}$, then $K^{\prime}(\varphi) x \in C_{\Delta}^{2}$ and

$$
\left\|\left(K^{\prime}(\varphi) x\right)^{(2)}\right\|_{\infty} \leq C_{1}\|x\|_{\infty} .
$$

Hence, by (2.19),

$$
\left\|\left(I-Q_{n}\right) K^{\prime}(\varphi) x\right\|_{\infty} \leq C_{4}\left\|\left(K^{\prime}(\varphi) x\right)^{(2)}\right\|_{\infty} h^{2} \leq C_{1} C_{4}\|x\|_{\infty} h^{2} .
$$

As a consequence,

$$
\left\|\left(I-Q_{n}\right) K^{\prime}(\varphi)\right\|=O\left(h^{2}\right) .
$$

Since $g \in C_{\Delta}^{\alpha}$, by (2.19),

$$
\left\|\left(I-Q_{n}\right) g\right\|_{\infty} \leq C_{4}\left\|g^{(\beta)}\right\|_{\infty} h^{\beta} .
$$

The required result then follows from the above two estimates.

The following lemma is crucial, and the proofs of many results which follow will be based on it.

Lemma 3.4. Let $g \in C_{\Delta}$. For a fixed $s \in[0,1]$, let $\ell_{s}(t)=\ell_{*}(s, t)$, $t \in[0,1]$. Then

$$
\left|K^{\prime}(\varphi)\left(I-Q_{n}\right) g(s)\right| \leq C_{10}\left(\sum_{j=1}^{n}\left\|\left(I-P_{n, j}\right)\left(\ell_{s} \delta_{j}^{r+1} g\right)\right\|_{\infty, \Delta_{j}}\right) h^{r+2},
$$

where $C_{10}$ is a constant independent of $n$.

Proof. For a fixed $s \in[0,1]$, we have

$$
K^{\prime}(\varphi)\left(I-Q_{n}\right) g(s)=\int_{0}^{1} \ell_{*}(s, t)\left(I-Q_{n}\right) g(t) d t
$$




$$
=\sum_{j=1}^{n} \int_{t_{j-1}}^{t_{j}} \ell_{s}(t)\left(I-Q_{n, j}\right) g(t) d t=\sum_{j=1}^{n}\left\langle\left(I-Q_{n, j}\right) g, \bar{\ell}_{s}\right\rangle_{j} .
$$

Hence, by Lemma 2.3,

$$
K^{\prime}(\varphi)\left(I-Q_{n}\right) g(s)=\sum_{j=1}^{n}\left\langle\left(I-P_{n, j}\right)\left(\ell_{s} \delta_{j}^{r+1} g\right), v_{j}\right\rangle_{j},
$$

where

$$
v_{j}(t)=\prod_{p=1}^{r+1}\left(t-\tau_{p}^{j}\right) .
$$

Using the fact that $P_{n, j}$ is the orthogonal projection, we obtain

$$
\begin{aligned}
\left|K^{\prime}(\varphi)\left(I-Q_{n}\right) g(s)\right| \leq & \sum_{j=1}^{n}\left|\left\langle\left(I-P_{n, j}\right)\left(\ell_{s} \delta_{j}^{r+1} g\right),\left(I-P_{n, j}\right) v_{j}\right\rangle_{j}\right| \\
\leq & \sum_{j=1}^{n}\left\|\left(I-P_{n, j}\right)\left(\ell_{s} \delta_{j}^{r+1} g\right)\right\|_{\infty, \Delta_{j}} \\
& \times\left\|\left(I-P_{n, j}\right) v_{j}\right\|_{\infty, \Delta_{j}} h_{j} .
\end{aligned}
$$

From (2.21),

$$
\left\|\left(I-P_{n, j}\right) v_{j}\right\|_{\infty, \Delta_{j}} \leq C_{5}\left\|\left(v_{j}\right)^{(r+1)}\right\|_{\infty} h_{j}^{r+1}=C_{5}(r+1) ! h_{j}^{r+1} .
$$

Hence,

$$
\left|K^{\prime}(\varphi)\left(I-Q_{n}\right) g(s)\right| \leq C_{5}(r+1) !\left(\sum_{j=1}^{n}\left\|\left(I-P_{n, j}\right)\left(\ell_{s} \delta_{j}^{r+1} g\right)\right\|_{\infty, \Delta_{j}}\right) h^{r+2},
$$

which completes the proof with $C_{10}=C_{5}(r+1)$ ! .

Below we prove two results which will be used in subsection 4.2 to obtain the order of convergence of the iterated modified projection solution.

Proposition 3.5. Let $r \geq 0$. Then

$$
\begin{aligned}
\left\|K^{\prime}(\varphi)\left(I-Q_{n}\right) K^{\prime}(\varphi)\right\| & =O\left(h^{r+1}\right), \\
\left\|K^{\prime}(\varphi)\left(I-Q_{n}\right) K^{\prime \prime}(\varphi)\right\| & =O\left(h^{r+1}\right),
\end{aligned}
$$




$$
\left\|K^{\prime}(\varphi)\left(I-Q_{n}\right) K^{(3)}(\varphi)\right\|=O\left(h^{r+1}\right) .
$$

Proof. Let $g \in C_{\Delta}$, and fix $s \in[0,1]$. By Lemma 3.4,

$$
\begin{aligned}
& \left|K^{\prime}(\varphi)\left(I-Q_{n}\right) K^{\prime}(\varphi) g(s)\right| \\
& \quad \leq C_{10}\left(\sum_{j=1}^{n}\left\|\left(I-P_{n, j}\right)\left(\ell_{s} \delta_{j}^{r+1} K^{\prime}(\varphi) g\right)\right\|_{\infty, \Delta_{j}}\right) h^{r+2} .
\end{aligned}
$$

Recall that

$$
\left(\delta_{j}^{r+1} K^{\prime}(\varphi) g\right)(t)=\left[\tau_{1}^{j}, \cdots, \tau_{r+1}^{j}, t\right] K^{\prime}(\varphi) g, \quad t \in \Delta_{j} .
$$

Hence, by Lemma 3.1,

$$
\left\|\delta_{j}^{r+1} K^{\prime}(\varphi) g\right\|_{\infty, \Delta_{j}}=\sup _{t \in \Delta_{j}}\left|\left(\delta_{j}^{r+1} K^{\prime}(\varphi) g\right)(t)\right| \leq C_{6}\|g\|_{\infty} .
$$

Thus,

$$
\left\|K^{\prime}(\varphi)\left(I-Q_{n}\right) K^{\prime}(\varphi) g\right\|_{\infty} \leq C_{6} C_{10}\left(1+\sup _{n, j}\left\|P_{n, j}\right\|\right)\left\|\ell_{*}\right\|_{\infty}\|g\|_{\infty} h^{r+1} .
$$

Since, by (2.20), $\sup _{n, j}\left\|P_{n, j}\right\|<\infty$, we obtain

$$
\left\|K^{\prime}(\varphi)\left(I-Q_{n}\right) K^{\prime}(\varphi)\right\|=\sup _{\|g\|_{\infty} \leq 1}\left\|K^{\prime}(\varphi)\left(I-Q_{n}\right) K^{\prime}(\varphi) g\right\|_{\infty}=O\left(h^{r+1}\right) .
$$

This completes the proof of (3.15). The proofs of (3.16) and of (3.17) are similar.

Proposition 3.6. Let $g \in C_{\Delta}^{\alpha}$ and $r \geq 0$. Then

$$
\left\|K^{\prime}(\varphi)\left(I-Q_{n}\right) K^{\prime}(\varphi)\left(I-Q_{n}\right) g\right\|_{\infty}=O\left(h^{\beta+r+2}\right) .
$$

Proof. Fix $s \in[0,1]$. By Lemma 3.4,

$$
\begin{aligned}
& \left|K^{\prime}(\varphi)\left(I-Q_{n}\right) K^{\prime}(\varphi)\left(I-Q_{n}\right) g(s)\right| \\
& \quad \leq C_{10}\left(\sum_{j=1}^{n}\left\|\left(I-P_{n, j}\right)\left(\ell_{s} \delta_{j}^{r+1} K^{\prime}(\varphi)\left(I-Q_{n}\right) g\right)\right\|_{\infty, \Delta_{j}}\right) h^{r+2} .
\end{aligned}
$$


Let $s \in \Delta_{i}=\left[t_{i-1}, t_{i}\right]$, and let $j \neq i$, Then $\ell_{s} \in C^{\alpha}\left(\Delta_{j}\right)$. Hence, by $(2.21)$,

$$
\begin{aligned}
& \left\|\left(I-P_{n, j}\right)\left(\ell_{s} \delta_{j}^{r+1} K^{\prime}(\varphi)\left(I-Q_{n}\right) g\right)\right\|_{\infty, \Delta_{j}} \\
& \leq C_{5} \|\left(\ell_{s}\right)^{\prime}\left(\delta_{j}^{r+1} K^{\prime}(\varphi)\left(I-Q_{n}\right) g\right) \\
& \quad+\ell_{s}\left(\delta_{j}^{r+1} K^{\prime}(\varphi)\left(I-Q_{n}\right) g\right)^{\prime} \|_{\infty, \Delta_{j}} h_{j} .
\end{aligned}
$$

By Lemma 3.1 and estimate (2.19), we obtain $\left\|\delta_{j}^{r+1} K^{\prime}(\varphi)\left(I-Q_{n}\right) g\right\|_{\infty, \Delta_{j}} \leq C_{6}\left\|\left(I-Q_{n}\right) g\right\|_{\infty, \Delta_{j}} \leq C_{6} C_{4}\left\|g^{(\beta)}\right\|_{\infty} h^{\beta}$.

Similarly, by Lemma 3.2 and estimate (2.19), we obtain

$$
\begin{aligned}
& \left\|\left(\delta_{j}^{r+1} K^{\prime}(\varphi)\left(I-Q_{n}\right) g\right)^{\prime}\right\|_{\infty, \Delta_{j}} \\
& =\sup _{t \in \Delta_{j}}\left|\left[\tau_{1}^{j}, \ldots, \tau_{r+1}^{j}, t, t\right] K^{\prime}(\varphi)\left(I-Q_{n}\right) g\right| \\
& \leq C_{9}\left\|\left(I-Q_{n}\right) g\right\|_{\infty, \Delta_{j}} \leq C_{9} C_{4}\left\|g^{(\beta)}\right\|_{\infty} h^{\beta} .
\end{aligned}
$$

Hence, for $j \neq i$,

$$
\begin{aligned}
& \left\|\left(I-P_{n, j}\right)\left(\ell_{s} \delta_{j}^{r+1} K^{\prime}(\varphi)\left(I-Q_{n}\right) g\right)\right\|_{\infty, \Delta_{j}} \\
& \quad \leq C_{4} C_{5}\left(C_{6}+C_{9}\right)\left\|g^{(\beta)}\right\|_{\infty} h^{\beta+1}=O\left(h^{\beta+1}\right) .
\end{aligned}
$$

On the other hand, by Lemma 3.1,

$$
\begin{aligned}
& \left\|\left(I-P_{n, i}\right)\left(\ell_{s} \delta_{i}^{r+1} K^{\prime}(\varphi)\left(I-Q_{n}\right) g\right)\right\|_{\infty, \Delta_{i}} \\
& \quad \leq\left(1+\left\|P_{n, i}\right\|\right)\left\|\ell_{*}\right\|_{\infty}\left\|\delta_{i}^{r+1} K^{\prime}(\varphi)\left(I-Q_{n}\right) g\right\|_{\infty, \Delta_{i}} \\
& \quad \leq C_{6}\left(1+\sup _{n}\left\|P_{n, i}\right\|\right)\left\|\ell_{*}\right\|_{\infty}\left\|\left(I-Q_{n}\right) g\right\|_{\infty, \Delta_{i}} \\
& \quad \leq C_{4} C_{6}\left(1+\sup _{n}\left\|P_{n, i}\right\|\right)\left\|\ell_{*}\right\|_{\infty}\left\|g^{(\beta)}\right\|_{\infty} h^{\beta}=O\left(h^{\beta}\right) .
\end{aligned}
$$

We thus obtain

$$
\begin{aligned}
& \left|K^{\prime}(\varphi)\left(I-Q_{n}\right) K^{\prime}(\varphi)\left(I-Q_{n}\right) g(s)\right| \\
& \leq C_{10}\left(\sum_{j=1 j \neq i}^{n}\left\|\left(I-P_{n, j}\right)\left(\ell_{s} \delta_{j}^{r+1} K^{\prime}(\varphi)\left(I-Q_{n}\right) g\right)\right\|_{\infty, \Delta_{j}}\right) h^{r+2} \\
& \quad+C_{10}\left\|\left(I-P_{n, i}\right)\left(\ell_{s} \delta_{i}^{r+1} K^{\prime}(\varphi)\left(I-Q_{n}\right) g\right)\right\|_{\infty, \Delta_{i}} h^{r+2}=O\left(h^{\beta+r+2}\right) .
\end{aligned}
$$

Since $s \in[0,1]$ is arbitrary, this completes the proof. 
In the case of $r=0$, since $\alpha \geq 2$, it follows that $\beta=1$. From the above proposition, we then obtain $\left\|K^{\prime}(\varphi)\left(I-Q_{n}\right) K^{\prime}(\varphi)\left(I-Q_{n}\right) g\right\|_{\infty}=$ $O\left(h^{3}\right)$. We now show that, if $\alpha \geq 4$, then the above order of convergence can be improved to $h^{4}$.

3.3. Interpolation at midpoints. Let $\mathcal{P}_{0, \Delta}$ be the space of piecewise constant functions with respect to the partition $\Delta$ defined in subsection 2.2 , and let

$$
\tau^{j}=\frac{t_{j-1}+t_{j}}{2}, \quad 1 \leq j \leq n,
$$

be the collocation points. Let $Q_{n}: C_{\Delta} \rightarrow \mathcal{P}_{0, \Delta}$ be the interpolatory projection defined as follows:

$$
Q_{n} x \in \mathcal{P}_{0, \Delta}, \quad\left(Q_{n} x\right)\left(\tau^{j}\right)=x\left(\tau^{j}\right), \quad 1 \leq j \leq n .
$$

The proof of the following Proposition consists of writing the Taylor series expansions for the kernel of the linear integral operator $K^{\prime}(\varphi)$ and for the function $K^{\prime}(\varphi)\left(I-Q_{n}\right) g$ about $\tau^{j}$ and using the fact that $\tau^{j}$ is the midpoint of $\left[t_{j-1}, t_{j}\right]$.

Proposition 3.7. Let $\alpha \geq 4$ and $g \in C_{\Delta}^{2}$. Let $Q_{n}$ be the interpolatory projection defined in (3.18). Then

$$
\left\|K^{\prime}(\varphi)\left(I-Q_{n}\right) K^{\prime}(\varphi)\left(I-Q_{n}\right) g\right\|_{\infty}=O\left(h^{4}\right)
$$

and

$$
\left\|K^{\prime}(\varphi)\left(I-Q_{n}\right) K^{\prime \prime}(\varphi)\left(Q_{n} \varphi-\varphi\right)^{2}\right\|_{\infty}=O\left(h^{4}\right) .
$$

Proof. Fix $s \in[0,1]$, and let $s \in \Delta_{i}=\left[t_{i-1}, t_{i}\right]$ for some $i$. Then

$$
\begin{aligned}
K^{\prime}(\varphi) & \left(I-Q_{n}\right) K^{\prime}(\varphi)\left(I-Q_{n}\right) g(s) \\
= & \sum_{j=1}^{i-1} \int_{t_{j-1}}^{t_{j}} \ell_{*}(s, t)\left(I-Q_{n, j}\right) K^{\prime}(\varphi)\left(I-Q_{n}\right) g(t) d t \\
& +\int_{t_{i-1}}^{t_{i}} \ell_{*}(s, t)\left(I-Q_{n, i}\right) K^{\prime}(\varphi)\left(I-Q_{n}\right) g(t) d t \\
& +\sum_{j=i+1}^{n} \int_{t_{j-1}}^{t_{j}} \ell_{*}(s, t)\left(I-Q_{n, j}\right) K^{\prime}(\varphi)\left(I-Q_{n}\right) g(t) d t .
\end{aligned}
$$


Case 1. $j \leq i-1$. If $t \in\left[t_{j-1}, t_{j}\right]$, then $t \leq s$, and hence

$$
\begin{aligned}
& \int_{t_{j-1}}^{t_{j}} \ell_{*}(s, t)\left(I-Q_{n, j}\right) K^{\prime}(\varphi)\left(I-Q_{n}\right) g(t) d t \\
= & \int_{t_{j-1}}^{t_{j}} \ell_{1, *}(s, t)\left[\left(K^{\prime}(\varphi)\left(I-Q_{n}\right) g\right)(t)-\left(K^{\prime}(\varphi)\left(I-Q_{n}\right) g\right)\left(\tau^{j}\right)\right] d t .
\end{aligned}
$$

Since $\alpha \geq 4$, it follows that $K^{\prime}(\varphi)\left(I-Q_{n}\right) g \in C_{\Delta}^{4}$. On writing the Taylor series expansions for $\ell_{1, *}(s, \cdot)$ and for $K^{\prime}(\varphi)\left(I-Q_{n}\right) g$ about $t=\tau^{j}$, we obtain

$$
\begin{aligned}
& \int_{t_{j-1}}^{t_{j}} \ell_{*}(s, t)\left(I-Q_{n, j}\right) K^{\prime}(\varphi)\left(I-Q_{n}\right) g(t) d t \\
& =\int_{t_{j-1}}^{t_{j}}\left[\ell_{1, *}\left(s, \tau^{j}\right)+\frac{\partial \ell_{1, *}}{\partial t}\left(s, \eta_{j}\right)\left(t-\tau^{j}\right)\right] \\
& \times\left[\sum_{p=1}^{3}\left(K^{\prime}(\varphi)\left(I-Q_{n}\right) g\right)^{(p)}\left(\tau^{j}\right) \frac{\left(t-\tau^{j}\right)^{p}}{p !}\right] d t \\
& \quad+\int_{t_{j-1}}^{t_{j}}\left[\ell_{1, *}\left(s, \tau^{j}\right)+\frac{\partial \ell_{1, *}}{\partial t}\left(s, \eta_{j}\right)\left(t-\tau^{j}\right)\right] \\
& \times\left[\left(K^{\prime}(\varphi)\left(I-Q_{n}\right) g\right)^{(4)}\left(\xi_{j}\right) \frac{\left(t-\tau^{j}\right)^{4}}{24}\right] d t
\end{aligned}
$$

where $\eta_{j}, \xi_{j} \in\left(t_{j-1}, t_{j}\right)$.

Since

$$
\int_{t_{j-1}}^{t_{j}}\left(t-\tau^{j}\right) d t=\int_{t_{j-1}}^{t_{j}}\left(t-\tau^{j}\right)^{3} d t=0
$$

we obtain

$$
\begin{aligned}
& \int_{t_{j-1}}^{t_{j}} \ell_{*}(s, t)\left(I-Q_{n, j}\right) K^{\prime}(\varphi)\left(I-Q_{n}\right) g(t) d t \\
& \quad=\ell_{1, *}\left(s, \tau^{j}\right)\left(K^{\prime}(\varphi)\left(I-Q_{n}\right) g\right)^{\prime \prime}\left(\tau^{j}\right) \int_{t_{j-1}}^{t_{j}} \frac{\left(t-\tau^{j}\right)^{2}}{2} d t \\
& \quad+\sum_{p=1}^{3}\left(K^{\prime}(\varphi)\left(I-Q_{n}\right) g\right)^{(p)}\left(\tau^{j}\right) \int_{t_{j-1}}^{t_{j}} \frac{\partial \ell_{1, *}}{\partial t}\left(s, \eta_{j}\right) \frac{\left(t-\tau^{j}\right)^{p+1}}{p !} d t
\end{aligned}
$$




$$
\begin{aligned}
& +\int_{t_{j-1}}^{t_{j}}\left[\ell_{1, *}\left(s, \tau^{j}\right)+\frac{\partial \ell_{1, *}}{\partial t}\left(s, \eta_{j}\right)\left(t-\tau^{j}\right)\right] \\
\times & {\left[\left(K^{\prime}(\varphi)\left(I-Q_{n}\right) g\right)^{(4)}\left(\xi_{j}\right) \frac{\left(t-\tau^{j}\right)^{4}}{24}\right] d t . }
\end{aligned}
$$

Thus,

$$
\begin{aligned}
& \left|\int_{t_{j-1}}^{t_{j}} \ell_{*}(s, t)\left(I-Q_{n, j}\right) K^{\prime}(\varphi)\left(I-Q_{n}\right) g(t) d t\right| \\
& \left.\quad \leq\left\|\ell_{1, *}\right\|_{\infty} \mid K^{\prime}(\varphi)\left(I-Q_{n}\right) g\right)^{\prime \prime}\left(\tau^{j}\right) \mid \frac{h_{j}^{3}}{24} \\
& \quad+\left\|D^{0,1} \ell_{1, *}\right\|_{\infty} \sum_{p=1}^{3}\left|\left(K^{\prime}(\varphi)\left(I-Q_{n}\right) g\right)^{(p)}\left(\tau^{j}\right)\right| \frac{h_{j}^{p+2}}{p !} \\
& \quad+\left(\left\|\ell_{1, *}\right\|_{\infty}+\left\|D^{0,1} \ell_{1, *}\right\|_{\infty} h_{j}\right) \\
& \quad \times\left\|\left(K^{\prime}(\varphi)\left(I-Q_{n}\right) g\right)^{(4)}\right\|_{\infty, \Delta_{j}} \frac{h_{j}^{5}}{24}
\end{aligned}
$$

where

$$
\begin{aligned}
\left\|\ell_{1, *}\right\|_{\infty} & =\sup \left\{\left|\ell_{1, *}(s, t)\right|:(s, t) \in \Omega_{1}\right\}, \\
\left\|D^{0,1} \ell_{1, *}\right\|_{\infty} & =\sup \left\{\left|D^{0,1} \ell_{1, *}(s, t)\right|:(s, t) \in \Omega_{1}\right\} .
\end{aligned}
$$

Since $\left(I-Q_{n}\right) g \in C_{\Delta}, \tau^{j} \notin \Delta$ and $\left(I-Q_{n}\right) g\left(\tau^{j}\right)=0$, using equations (2.10) and (2.11), we obtain

$$
\begin{aligned}
\left(K^{\prime}(\varphi)\left(I-Q_{n}\right) g\right)^{\prime}\left(\tau^{j}\right)= & \int_{0}^{\tau^{j}} \frac{\partial \ell_{1, *}}{\partial s}\left(\tau^{j}, t\right)\left(I-Q_{n}\right) g(t) d t \\
& +\int_{\tau^{j}}^{1} \frac{\partial \ell_{2, *}}{\partial s}\left(\tau^{j}, t\right)\left(I-Q_{n}\right) g(t) d t
\end{aligned}
$$

and

$$
\begin{aligned}
\left(K^{\prime}(\varphi)\left(I-Q_{n}\right) g\right)^{\prime \prime}\left(\tau^{j}\right)= & \int_{0}^{\tau^{j}} \frac{\partial^{2} \ell_{1, *}}{\partial s^{2}}\left(\tau^{j}, t\right)\left(I-Q_{n}\right) g(t) d t \\
& +\int_{\tau^{j}}^{1} \frac{\partial^{2} \ell_{2, *}}{\partial s^{2}}\left(\tau^{j}, t\right)\left(I-Q_{n}\right) g(t) d t
\end{aligned}
$$


Since $\ell_{*} \in \mathcal{G}(\alpha, 0)$ and $g \in C_{\Delta}^{2}$, using the technique used in [5, Theorem $15]$, we obtain the following estimates.

$$
\begin{aligned}
\left|\left(K^{\prime}(\varphi)\left(I-Q_{n}\right) g\right)^{\prime}\left(\tau^{j}\right)\right| & =O\left(h^{2}\right), \\
\left|\left(K^{\prime}(\varphi)\left(I-Q_{n}\right) g\right)^{\prime \prime}\left(\tau^{j}\right)\right| & =O\left(h^{2}\right) .
\end{aligned}
$$

Using (2.14), we deduce the following estimates:

$$
\begin{aligned}
\left|\left(K^{\prime}(\varphi)\left(I-Q_{n}\right) g\right)^{(3)}\left(\tau^{j}\right)\right| & \leq C_{3}\left(\left\|\left(I-Q_{n}\right) g\right\|_{\infty}+\left\|\left(\left(I-Q_{n}\right) g\right)^{\prime}\right\|_{\infty}\right) \\
& \leq C_{3}\left(1+\left\|\left.Q_{n}\right|_{C_{\Delta}}\right\|\right)\left(\|g\|_{\infty}+\left\|g^{\prime}\right\|_{\infty}\right)
\end{aligned}
$$

and

$$
\begin{aligned}
\left\|\left(K^{\prime}(\varphi)\left(I-Q_{n}\right) u\right)^{(4)}\right\|_{\infty, \Delta_{j}} & \\
& \leq C_{3}\left(1+\left\|\left.Q_{n}\right|_{C_{\Delta}}\right\|\right)\left(\|g\|_{\infty}+\left\|g^{\prime}\right\|_{\infty}+\left\|g^{\prime \prime}\right\|_{\infty}\right) .
\end{aligned}
$$

Since, by (2.16), $\sup _{n}\left\|\left.Q_{n}\right|_{C_{\Delta}}\right\|<\infty$, it follows that the above two quantities are bounded by a constant independent of $n$. Thus, using estimates (3.22) and (3.23), we obtain

$$
\left|\int_{t_{j-1}}^{t_{j}} \ell_{*}(s, t)\left(I-Q_{n}\right) K^{\prime}(\varphi)\left(I-Q_{n}\right) u(t) d t\right|=O\left(h^{5}\right) .
$$

Case 2. $j=i$. In this case,

$$
\begin{aligned}
& \int_{t_{i-1}}^{t_{i}} \ell_{*}(s, t)\left(I-Q_{n, i}\right) K^{\prime}(\varphi)\left(I-Q_{n}\right) g(t) d t \\
& =\int_{t_{i-1}}^{t_{i}} \ell_{*}(s, t)\left[K^{\prime}(\varphi)\left(I-Q_{n}\right) g(t)-K^{\prime}(\varphi)\left(I-Q_{n}\right) g\left(\tau^{i}\right)\right] d t \\
& =\int_{t_{i-1}}^{t_{i}} \ell_{*}(s, t)\left(K^{\prime}(\varphi)\left(I-Q_{n}\right) g\right)^{\prime}\left(\xi_{i}\right)\left(t-\tau^{i}\right) d t,
\end{aligned}
$$

where $\xi_{i} \in\left(t_{i-1}, t_{i}\right)$.

By [5, Theorem 15],

$$
\left.\left|\left(K^{\prime}(\varphi)\left(I-Q_{n}\right) g\right)^{\prime}\left(\xi_{i}\right)\right| \leq \sup _{t \in\left[t_{i-1}, t_{i}\right]} \mid K^{\prime}(\varphi)\left(I-Q_{n}\right) g\right)^{\prime}(t) \mid=O\left(h^{2}\right) .
$$


Hence,

$$
\left|\int_{t_{i-1}}^{t_{i}} \ell_{*}(\mu, t)\left(I-Q_{n}\right) K^{\prime}(\varphi)\left(I-Q_{n}\right) g(t) d t\right|=O\left(h^{4}\right) .
$$

Case 3. $j \geq i+1$.

Note that, if $t \in\left[t_{j-1}, t_{j}\right]$, then $t \geq s$. Hence, $\ell_{*}(s, t)=\ell_{2, *}(s, t)$. As in Case 1, it follows that

$$
\left|\int_{t_{j-1}}^{t_{j}} \ell_{*}(s, t)\left(I-Q_{n}\right) K^{\prime}(\varphi)\left(I-Q_{n}\right) u(t) d t\right|=O\left(h^{5}\right) .
$$

Estimate (3.19) follows from (3.21), (3.24), (3.25) and (3.26). The proof of (3.20) is similar.

4. Orders of convergence. We now obtain the orders of convergence of the modified projection solution $\varphi_{n}^{M}$ and of the iterated modified projection solution $\widetilde{\varphi}_{n}^{M}$.

4.1. Modified projection method. We first prove the following result that is needed in the proof of the Theorem 4.2, which is the main theorem in this section.

Lemma 4.1. Let $r \geq 0$. Then

$$
\left\|\left(I-Q_{n}\right)\left[K\left(Q_{n} \varphi\right)-K(\varphi)-K^{\prime}(\varphi)\left(Q_{n} \varphi-\varphi\right)\right]\right\|_{\infty}=O\left(h^{2 \beta+1}\right) .
$$

Proof. Let $n_{0}$ be a positive integer such that $n \geq n_{0}$ implies that $Q_{n} \varphi \in \mathcal{B}\left(\varphi, \delta_{0}\right)$. Then, by Taylor's theorem,

$$
\begin{aligned}
K\left(Q_{n} \varphi\right)-K(\varphi)-K^{\prime}(\varphi)( & \left.Q_{n} \varphi-\varphi\right) \\
& =\frac{1}{2} K^{\prime \prime}(\varphi)\left(Q_{n} \varphi-\varphi\right)^{2}+R\left(Q_{n} \varphi-\varphi\right),
\end{aligned}
$$

where

$$
\begin{aligned}
& R\left(Q_{n} \varphi-\varphi\right)(s) \\
= & \int_{0}^{1}\left[K^{(3)}\left(\varphi+\theta\left(Q_{n} \varphi-\varphi\right)\right)\left(Q_{n} \varphi-\varphi\right)^{3}\right](s) \frac{(1-\theta)^{2}}{2} d \theta, \quad s \in[0,1] .
\end{aligned}
$$

Recall from (2.7) that 


$$
\begin{aligned}
{\left[K^{(3)}(\varphi+\right.} & \left.\left.\theta\left(Q_{n} \varphi-\varphi\right)\right)\left(Q_{n} \varphi-\varphi\right)^{3}\right](s) \\
& =\int_{0}^{1} \frac{\partial^{3} \kappa\left(s, t, \varphi(t)+\theta\left(Q_{n} \varphi-\varphi\right)(t)\right)}{\partial u^{3}}\left(Q_{n} \varphi-\varphi\right)^{3}(t) d t
\end{aligned}
$$

Then

$$
\begin{aligned}
\mid\left[K^{(3)}\left(\varphi+\theta\left(Q_{n} \varphi-\varphi\right)\right)\right. & \left.\left(Q_{n} \varphi-\varphi\right)^{3}\right](s) \mid \\
\leq & \sup _{\substack{s, t \in[0,1] \\
|u| \leq\|\varphi\|_{\infty}+\delta_{0}}}\left|\frac{\partial^{3} \kappa}{\partial u^{3}}(s, t, u)\right|\left\|Q_{n} \varphi-\varphi\right\|_{\infty}^{3} .
\end{aligned}
$$

Hence, using the notation introduced in (2.27),

$$
\left\|K^{(3)}\left(\varphi+\theta\left(Q_{n} \varphi-\varphi\right)\right)\left(Q_{n} \varphi-\varphi\right)^{3}\right\|_{\infty} \leq M_{2}\left\|Q_{n} \varphi-\varphi\right\|_{\infty}^{3} .
$$

As a consequence,

$$
\left\|R\left(Q_{n} \varphi-\varphi\right)\right\|_{\infty} \leq \frac{1}{6} M_{2}\left\|Q_{n} \varphi-\varphi\right\|_{\infty}^{3} .
$$

Note that

$$
\begin{aligned}
\left(I-Q_{n}\right)\left[K\left(Q_{n} \varphi\right)-K(\varphi)-K^{\prime}(\varphi)\left(Q_{n} \varphi-\varphi\right)\right] \\
=\frac{1}{2}\left(I-Q_{n}\right) K^{\prime \prime}(\varphi)\left(Q_{n} \varphi-\varphi\right)^{2} \\
\quad+\left(I-Q_{n}\right) R\left(Q_{n} \varphi-\varphi\right) .
\end{aligned}
$$

By (2.4), $\varphi \in C^{\alpha}[0,1]$. Hence, using (2.19), we obtain

$$
\left\|Q_{n} \varphi-\varphi\right\|_{\infty} \leq C_{4}\left\|\varphi^{(\beta)}\right\|_{\infty} h^{\beta} .
$$

Since $Q_{n} \varphi-\varphi \in C_{\Delta}$, it follows that $K^{\prime \prime}(\varphi)\left(Q_{n} \varphi-\varphi\right)^{2} \in C_{\Delta}^{2}$. Hence,

$$
\left\|\left(I-Q_{n}\right) K^{\prime \prime}(\varphi)\left(Q_{n} \varphi-\varphi\right)^{2}\right\|_{\infty} \leq C_{4}\left\|\left(K^{\prime \prime}(\varphi)\left(Q_{n} \varphi-\varphi\right)^{2}\right)^{\prime}\right\|_{\infty} h .
$$

By (2.13),

$$
\left\|\left(K^{\prime \prime}(\varphi)\left(Q_{n} \varphi-\varphi\right)^{2}\right)^{\prime}\right\|_{\infty} \leq C_{2}\left\|Q_{n} \varphi-\varphi\right\|_{\infty}^{2} .
$$

Hence, using (4.2), we obtain

$$
\left\|\left(I-Q_{n}\right) K^{\prime \prime}(\varphi)\left(Q_{n} \varphi-\varphi\right)^{2}\right\|_{\infty} \leq C_{2}\left(C_{4}\right)^{3}\left\|\varphi^{(\beta)}\right\|_{\infty}^{2} h^{2 \beta+1} .
$$


On the other hand,

$$
\left\|\left(I-Q_{n}\right) R\left(Q_{n} \varphi-\varphi\right)\right\|_{\infty} \leq \frac{1}{6}\left(1+\left\|\left.Q_{n}\right|_{C_{\Delta}}\right\|\right) M_{2}\left\|Q_{n} \varphi-\varphi\right\|_{\infty}^{3} .
$$

Since, by $(2.16), \sup _{n}\left\|\left.Q_{n}\right|_{C_{\Delta}}\right\|<\infty$, it follows that

$$
\left\|\left(I-Q_{n}\right) R\left(Q_{n} \varphi-\varphi\right)\right\|_{\infty}=O\left(h^{3 \beta}\right) .
$$

As $\beta \geq 1$, the required estimate follows from (4.1), (4.3) and (4.4).

Theorem 4.2. Let $\alpha \geq 2$, and let the kernel $\kappa$ of the Urysohn integral operator $K$ defined by (2.1) satisfy the assumptions $\left(H_{1}\right),\left(H_{2}\right)$ and $\left(H_{3}\right)$ in subsection 2.1. Let $f \in C^{\alpha}[0,1]$ and $\varphi$ be the unique solution of (2.3). Assume that 1 is not an eigenvalue of $K^{\prime}(\varphi)$. Let $r \geq 0$ and $\mathcal{P}_{r, \Delta}$ be the space of piecewise polynomials of degree $\leq r$ with respect to a quasiuniform partition defined in subsection 2.2. Let $Q_{n}: C_{\Delta} \rightarrow \mathcal{P}_{r, \Delta}$ be the interpolatory projection defined by (2.15). Let $\varphi_{n}^{M}$ be the unique solution of (2.24) in a neighborhood $B\left(\varphi, \delta_{0}\right)$ of $\varphi$. Then

$$
\left\|\varphi_{n}^{M}-\varphi\right\|_{\infty}=O\left(h^{\beta+2}\right) .
$$

Proof. Using Theorem 2.5 and Proposition 2.6 we get

$$
\begin{aligned}
\left\|\varphi_{n}^{M}-\varphi\right\|_{\infty} & \leq 2\left\|\left(I-\left(K_{n}^{M}\right)^{\prime}(\varphi)\right)^{-1}\left[K(\varphi)-K_{n}^{M}(\varphi)\right]\right\|_{\infty} \\
& \leq 4\left\|\left(I-K^{\prime}(\varphi)\right)^{-1}\right\|\left\|\left[K(\varphi)-K_{n}^{M}(\varphi)\right]\right\|_{\infty} .
\end{aligned}
$$

Consider

$$
\begin{aligned}
K(\varphi)-K_{n}^{M}(\varphi)= & \left(I-Q_{n}\right)\left(K(\varphi)-K\left(Q_{n} \varphi\right)\right) \\
= & -\left(I-Q_{n}\right)\left[K\left(Q_{n} \varphi\right)-K(\varphi)-K^{\prime}(\varphi)\left(Q_{n} \varphi-\varphi\right)\right] \\
& +\left(I-Q_{n}\right) K^{\prime}(\varphi)\left(I-Q_{n}\right) \varphi .
\end{aligned}
$$

Note that $\varphi \in C^{\alpha}[0,1]$. By Lemma 4.1,

$$
\left\|\left(I-Q_{n}\right)\left[K\left(Q_{n} \varphi\right)-K(\varphi)-K^{\prime}(\varphi)\left(Q_{n} \varphi-\varphi\right)\right]\right\|_{\infty}=O\left(h^{2 \beta+1}\right)
$$

and, by Proposition 3.3,

$$
\left\|\left(I-Q_{n}\right) K^{\prime}(\varphi)\left(I-Q_{n}\right) \varphi\right\|_{\infty}=O\left(h^{\beta+2}\right) .
$$

Since $\beta=\min \{\alpha, r+1\} \geq 1$, the desired estimate follows. 
4.2. Iterated modified projection method. In this section, we prove our main result. We show that the order of convergence in the iterated modified method is higher than those in the collocation and in the iterated collocation/modified projection methods. We prove below a series of results which are needed in the proof of our main theorem. From now on, we assume that $n_{0}$ is a positive integer such that $n \geq n_{0}$ implies that $Q_{n} \varphi \in \mathcal{B}\left(\varphi, \delta_{0}\right)$.

Lemma 4.3. If $\alpha \geq 4$ and $r=0$, then

$$
\left\|K^{\prime}(\varphi)\left(I-Q_{n}\right)\left[K\left(Q_{n} \varphi\right)-K(\varphi)-K^{\prime}(\varphi)\left(Q_{n} \varphi-\varphi\right)\right]\right\|_{\infty}=O\left(h^{4}\right) .
$$

If $\alpha \geq 2$ and $r \geq 1$, then

$$
\left\|K^{\prime}(\varphi)\left(I-Q_{n}\right)\left[K\left(Q_{n} \varphi\right)-K(\varphi)-K^{\prime}(\varphi)\left(Q_{n} \varphi-\varphi\right)\right]\right\|_{\infty}=O\left(h^{3 \beta}\right) .
$$

Proof. By Taylor's theorem,

$$
\begin{aligned}
& K\left(Q_{n} \varphi\right)-K(\varphi)-K^{\prime}(\varphi)\left(Q_{n} \varphi-\varphi\right) \\
& \quad=\frac{1}{2} K^{\prime \prime}(\varphi)\left(Q_{n} \varphi-\varphi\right)^{2}+\frac{1}{6} K^{(3)}(\varphi)\left(Q_{n} \varphi-\varphi\right)^{3}+R\left(Q_{n} \varphi-\varphi\right),
\end{aligned}
$$

where

$$
\begin{aligned}
& R\left(Q_{n} \varphi-\varphi\right)(s) \\
= & \int_{0}^{1}\left[K^{(4)}\left(\varphi+\theta\left(Q_{n} \varphi-\varphi\right)\right)\left(Q_{n} \varphi-\varphi\right)^{4}\right](s) \frac{(1-\theta)^{3}}{6} d \theta, \quad s \in[0,1] .
\end{aligned}
$$

Recall from (2.8) that

$$
\begin{aligned}
{\left[K^{(4)}(\varphi+\right.} & \left.\left.\theta\left(Q_{n} \varphi-\varphi\right)\right)\left(Q_{n} \varphi-\varphi\right)^{4}\right](s) \\
& =\int_{0}^{1} \frac{\partial^{4} \kappa\left(s, t, \varphi(t)+\theta\left(Q_{n} \varphi-\varphi\right)(t)\right)}{\partial u^{4}}\left(Q_{n} \varphi-\varphi\right)^{4}(t) d t
\end{aligned}
$$

Then

$$
\begin{aligned}
\mid\left[K^{(4)}\left(\varphi+\theta\left(Q_{n} \varphi-\varphi\right)\right)\right. & \left.\left(Q_{n} \varphi-\varphi\right)^{4}\right](s) \mid \\
\leq & \sup _{\substack{s, t \in[0,1] \\
|u| \leq\|\varphi\|_{\infty}+\delta_{0}}}\left|\frac{\partial^{4} \kappa}{\partial u^{4}}(s, t, u)\right|\left\|Q_{n} \varphi-\varphi\right\|_{\infty}^{4} .
\end{aligned}
$$


Hence, using the notation introduced in (2.28),

$$
\left\|K^{(4)}\left(\varphi+\theta\left(Q_{n} \varphi-\varphi\right)\right)\left(Q_{n} \varphi-\varphi\right)^{4}\right\|_{\infty} \leq M_{3}\left\|Q_{n} \varphi-\varphi\right\|_{\infty}^{4} .
$$

As a consequence,

$$
\left\|R\left(Q_{n} \varphi-\varphi\right)\right\|_{\infty} \leq \frac{1}{24} M_{3}\left\|Q_{n} \varphi-\varphi\right\|_{\infty}^{4} .
$$

Note that

$$
\begin{aligned}
K^{\prime}(\varphi)\left(I-Q_{n}\right)\left[K\left(Q_{n} \varphi\right)-K(\varphi)-K^{\prime}(\varphi)\left(Q_{n} \varphi-\varphi\right)\right] \\
=\frac{1}{2} K^{\prime}(\varphi)\left(I-Q_{n}\right) K^{\prime \prime}(\varphi)\left(Q_{n} \varphi-\varphi\right)^{2} \\
+\frac{1}{6} K^{\prime}(\varphi)\left(I-Q_{n}\right) K^{(3)}(\varphi)\left(Q_{n} \varphi-\varphi\right)^{3} \\
\quad+K^{\prime}(\varphi)\left(I-Q_{n}\right) R\left(Q_{n} \varphi-\varphi\right) .
\end{aligned}
$$

Recall from (4.2) that $\left\|Q_{n} \varphi-\varphi\right\|_{\infty}=O\left(h^{\beta}\right)$. If $r=0$, then, by Proposition 3.7,

$$
\left\|K^{\prime}(\varphi)\left(I-Q_{n}\right) K^{\prime \prime}(\varphi)\left(Q_{n} \varphi-\varphi\right)^{2}\right\|_{\infty}=O\left(h^{4}\right),
$$

whereas if $r \geq 1$, then, by Proposition 3.5,

$$
\begin{aligned}
\| K^{\prime}(\varphi) & \left(I-Q_{n}\right) K^{\prime \prime}(\varphi)\left(Q_{n} \varphi-\varphi\right)^{2} \|_{\infty} \\
& \leq\left\|K^{\prime}(\varphi)\left(I-Q_{n}\right) K^{\prime \prime}(\varphi)\right\|\left\|Q_{n} \varphi-\varphi\right\|_{\infty}^{2}=O\left(h^{2 \beta+r+1}\right)
\end{aligned}
$$

and

$$
\left\|K^{\prime}(\varphi)\left(I-Q_{n}\right) K^{(3)}(\varphi)\left(Q_{n} \varphi-\varphi\right)^{3}\right\|_{\infty}=O\left(h^{3 \beta+r+1}\right) .
$$

Since, by $(2.16), \sup _{n}\left\|\left.Q_{n}\right|_{C_{\Delta}}\right\|<\infty$, we get

$$
\begin{aligned}
& \left\|K^{\prime}(\varphi)\left(I-Q_{n}\right) R\left(Q_{n} \varphi-\varphi\right)\right\|_{\infty} \\
& \quad \leq\left\|K^{\prime}(\varphi)\right\|\left(1+\sup _{n} \|\left. Q_{n}\right|_{C_{\Delta}}\right)\left\|R\left(Q_{n} \varphi-\varphi\right)\right\|_{\infty}=O\left(h^{4 \beta}\right) .
\end{aligned}
$$

The required estimates follow from (4.6)-(4.10).

Lemma 4.4. If $\alpha \geq 4$ and $r=0$, then

$$
\left\|K^{\prime}(\varphi)\left[K(\varphi)-K_{n}^{M}(\varphi)\right]\right\|_{\infty}=O\left(h^{4}\right),
$$


and, if $\alpha \geq 2$ and $r \geq 1$, then

$$
\left\|K^{\prime}(\varphi)\left[K(\varphi)-K_{n}^{M}(\varphi)\right]\right\|_{\infty}=O\left(h^{2 \beta+1}\right) .
$$

Proof. Using the expression (4.5) for $K(\varphi)-K_{n}^{M}(\varphi)$, we deduce that

$$
\begin{aligned}
& \left\|K^{\prime}(\varphi)\left[K(\varphi)-K_{n}^{M}(\varphi)\right]\right\|_{\infty} \\
& \quad \leq\left\|K^{\prime}(\varphi)\left(I-Q_{n}\right)\left[K\left(Q_{n} \varphi\right)-K(\varphi)-K^{\prime}(\varphi)\left(Q_{n} \varphi-\varphi\right)\right]\right\|_{\infty} \\
& \quad+\left\|K^{\prime}(\varphi)\left(I-Q_{n}\right) K^{\prime}(\varphi)\left(I-Q_{n}\right) \varphi\right\|_{\infty} .
\end{aligned}
$$

The desired estimates follow from Proposition 3.6, Proposition 3.7 and Lemma 4.3.

Lemma 4.5. Let $\alpha \geq 2$ and $r \geq 0$. Then

$$
\left\|K^{\prime}(\varphi)\left(\left(K_{n}^{M}\right)^{\prime}(\varphi)-K^{\prime}(\varphi)\right)\left(\varphi-\varphi_{n}^{M}\right)\right\|_{\infty}=O\left(h^{2 \beta+2}\right) .
$$

Proof. Note that

$$
\begin{aligned}
& K^{\prime}(\varphi)\left(\left(K_{n}^{M}\right)^{\prime}(\varphi)-K^{\prime}(\varphi)\right) \\
& \quad=-K^{\prime}(\varphi)\left(I-Q_{n}\right) K^{\prime}(\varphi)+K^{\prime}(\varphi)\left(I-Q_{n}\right) K^{\prime}\left(Q_{n} \varphi\right) Q_{n} .
\end{aligned}
$$

By Proposition 3.5,

$$
\left\|K^{\prime}(\varphi)\left(I-Q_{n}\right) K^{\prime}(\varphi)\right\|=O\left(h^{r+1}\right) .
$$

On the other hand,

$$
\begin{aligned}
& K^{\prime}(\varphi)\left(I-Q_{n}\right) K^{\prime}\left(Q_{n} \varphi\right) Q_{n} \\
= & K^{\prime}(\varphi)\left(I-Q_{n}\right)\left(K^{\prime}\left(Q_{n} \varphi\right)-K^{\prime}(\varphi)\right) Q_{n}+K^{\prime}(\varphi)\left(I-Q_{n}\right) K^{\prime}(\varphi) Q_{n} .
\end{aligned}
$$

Since $K^{\prime}$ is Lipschitz in $\mathcal{B}\left(\varphi, \delta_{0}\right)$, by $(2.2)$,

$$
\left\|K^{\prime}\left(Q_{n} \varphi\right)-K^{\prime}(\varphi)\right\| \leq \gamma\left\|Q_{n} \varphi-\varphi\right\|_{\infty} .
$$

Hence, by (2.16) and (4.2),

$$
\begin{aligned}
& \left\|K^{\prime}(\varphi)\left(I-Q_{n}\right)\left(K^{\prime}\left(Q_{n} \varphi\right)-K^{\prime}(\varphi)\right) Q_{n}\right\| \\
& \leq\left\|K^{\prime}(\varphi)\right\|\left(\left\|\left.Q_{n}\right|_{C_{\Delta}}\right\|+\left\|\left.Q_{n}\right|_{C_{\Delta}}\right\|^{2}\right) \gamma\left\|Q_{n} \varphi-\varphi\right\|_{\infty}=O\left(h^{\beta}\right) .
\end{aligned}
$$

Using (4.11) and (4.12), we deduce that

$$
\left\|K^{\prime}(\varphi)\left(\left(K_{n}^{M}\right)^{\prime}(\varphi)-K^{\prime}(\varphi)\right)\right\|=O\left(h^{\beta}\right) .
$$


Since, by Theorem 4.2, $\left\|\varphi_{n}^{M}-\varphi\right\|_{\infty}=O\left(h^{\beta+2}\right)$, the desired estimate follows.

We now prove our main result.

Theorem 4.6. Let $\alpha \geq 2$, and let the kernel $\kappa$ of the Urysohn integral operator $K$ defined by (2.1) satisfy assumptions $\left(H_{1}\right),\left(H_{2}\right)$ and $\left(H_{3}\right)$ in subsection 2.1. Let $f \in C^{\alpha}[0,1]$ and $\varphi$ be the unique solution of $(2.3)$. Assume that 1 is not an eigenvalue of $K^{\prime}(\varphi)$. Let $r \geq 0$ and $\mathcal{P}_{r, \Delta}$ be the space of piecewise polynomials of degree $\leq r$ with respect to a quasi-uniform partition defined in subsection 2.2. Let $Q_{n}: C_{\Delta} \rightarrow \mathcal{P}_{r, \Delta}$ be the interpolatory projection defined by (2.15). Let $\varphi_{n}^{M}$ be the unique solution of (2.24) in a neighborhood $B\left(\varphi, \delta_{0}\right)$ of $\varphi$ and $\widetilde{\varphi}_{n}^{M}$ be defined by $(2.26)$.

If $\alpha \geq 4$ and $r=0$, then

$$
\left\|\widetilde{\varphi}_{n}^{M}-\varphi\right\|_{\infty}=O\left(h^{4}\right) .
$$

If $\alpha \geq 2$ and $r \geq 1$, then

$$
\left\|\widetilde{\varphi}_{n}^{M}-\varphi\right\|_{\infty}=O\left(h^{2 \beta+1}\right) .
$$

Proof. Since $\varphi=K(\varphi)+f$ and $\widetilde{\varphi}_{n}^{M}=K\left(\varphi_{n}^{M}\right)+f$, we obtain

$$
\widetilde{\varphi}_{n}^{M}-\varphi=K\left(\varphi_{n}^{M}\right)-K(\varphi) .
$$

By Taylor's theorem,

$$
K\left(\varphi_{n}^{M}\right)-K(\varphi)=K^{\prime}(\varphi)\left(\varphi_{n}^{M}-\varphi\right)+R\left(\varphi_{n}^{M}-\varphi\right)
$$

with

$$
\begin{aligned}
& \left(R\left(\varphi_{n}^{M}-\varphi\right)\right)(s) \\
& \quad=\int_{0}^{1}(1-\theta)\left[K^{\prime \prime}\left(\varphi+\theta\left(\varphi_{n}^{M}-\varphi\right)\right)\left(\varphi_{n}^{M}-\varphi\right)^{2}\right](s) d \theta, \quad s \in[0,1] .
\end{aligned}
$$

Since

$$
\begin{aligned}
& \left(K^{\prime \prime}\left(\varphi+\theta\left(\varphi_{n}^{M}-\varphi\right)\right)\left(\varphi_{n}^{M}-\varphi\right)^{2}\right)(s) \\
& \quad \int_{0}^{1} \frac{\partial^{2} \kappa}{\partial u^{2}}\left(s, t, \varphi(t)+\theta\left(\varphi_{n}^{M}(t)-\varphi(t)\right)\left(\varphi_{n}^{M}-\varphi\right)^{2} d t\right.
\end{aligned}
$$


we obtain

$$
\begin{aligned}
&\left|\left(K^{\prime \prime}\left(\varphi+\theta\left(\varphi_{n}^{M}-\varphi\right)\right)\left(\varphi_{n}^{M}-\varphi\right)^{2}\right)(s)\right| \\
& \leq \sup _{\substack{s, t \in[0,1] \\
|u| \leq\|\varphi\|_{\infty}+\delta_{0}}}\left|\frac{\partial^{2} \kappa}{\partial u^{2}}(s, t, u)\right|\left\|\varphi_{n}^{M}-\varphi\right\|^{2}
\end{aligned}
$$

Hence, using the notation introduced in (2.27),

$$
\left\|\left(K^{\prime \prime}\left(\varphi+\theta\left(\varphi_{n}^{M}-\varphi\right)\right)\left(\varphi_{n}^{M}-\varphi\right)^{2}\right)\right\|_{\infty} \leq M_{1}\left\|\varphi_{n}^{M}-\varphi\right\|^{2} .
$$

Since, by Theorem 4.2, $\left\|\varphi_{n}^{M}-\varphi\right\|_{\infty}=O\left(h^{\beta+2}\right)$, it follows that

$$
\left\|R\left(\varphi_{n}^{M}-\varphi\right)\right\|_{\infty}=O\left(h^{2 \beta+4}\right) .
$$

From [8, Theorem 3.5],

$$
\begin{aligned}
& K^{\prime}(\varphi)\left(\varphi_{n}^{M}-\varphi\right) \\
= & -\left(I-K^{\prime}(\varphi)\right)^{-1} K^{\prime}(\varphi)\left[K(\varphi)-K^{\prime}(\varphi) \varphi-K_{n}^{M}\left(\varphi_{n}^{M}\right)+K^{\prime}(\varphi) \varphi_{n}^{M}\right] .
\end{aligned}
$$

We write

$$
\begin{aligned}
& K^{\prime}(\varphi)\left(\varphi_{n}^{M}-\varphi\right) \\
& \quad=-\left(I-K^{\prime}(\varphi)\right)^{-1} K^{\prime}(\varphi)\left[K(\varphi)-K_{n}^{M}(\varphi)\right]+\left(I-K^{\prime}(\varphi)\right)^{-1} K^{\prime}(\varphi) \\
& \quad\left[K_{n}^{M}\left(\varphi_{n}^{M}\right)-K_{n}^{M}(\varphi)-\left(K_{n}^{M}\right)^{\prime}(\varphi)\left(\varphi_{n}^{M}-\varphi\right)\right] \\
& \quad+\left(I-K^{\prime}(\varphi)\right)^{-1} K^{\prime}(\varphi)\left[\left(\left(K_{n}^{M}\right)^{\prime}(\varphi)-K^{\prime}(\varphi)\right)\left(\varphi_{n}^{M}-\varphi\right)\right] .
\end{aligned}
$$

By Lemma 4.4, if $r=0$, then the first term in the above expression is of the order of $h^{4}$ and, if $r \geq 1$, then it is of the order of $h^{2 \beta+1}$. From [8, Lemma 3.3],

$$
\left\|K_{n}^{M}\left(\varphi_{n}^{M}\right)-K_{n}^{M}(\varphi)-\left(K_{n}^{M}\right)^{\prime}(\varphi)\left(\varphi_{n}^{M}-\varphi\right)\right\|_{\infty}=O\left(\left\|\varphi_{n}^{M}-\varphi\right\|_{\infty}^{2}\right) .
$$

Hence, the second term is of the order of $h^{2 \beta+4}$. Lastly, by Lemma 4.5, the third term is of the order of $h^{2 \beta+2}$. Thus, if $r=0$, then

$$
\left\|K^{\prime}(\varphi)\left(\varphi_{n}^{M}-\varphi\right)\right\|_{\infty}=O\left(h^{4}\right)
$$

and if $r \geq 1$, then

$$
\left\|K^{\prime}(\varphi)\left(\varphi_{n}^{M}-\varphi\right)\right\|_{\infty}=O\left(h^{2 \beta+1}\right) .
$$


It follows from (4.15)-(4.19) that, if $r=0$, then

$$
\left\|\widetilde{\varphi}_{n}^{M}-\varphi\right\|_{\infty}=\left\|K\left(\varphi_{n}^{M}\right)-K(\varphi)\right\|_{\infty}=O\left(h^{4}\right),
$$

and, if $r \geq 1$, then

$$
\left\|\widetilde{\varphi}_{n}^{M}-\varphi\right\|_{\infty}=O\left(h^{2 \beta+1}\right),
$$

which completes the proof.

Remark 4.7. First consider the case when $r=0$. If $\alpha \geq 2$, then recall from (2.22) and (2.23) that

$$
\left\|\varphi_{n}^{C}-\varphi\right\|_{\infty}=O(h), \quad\left\|\varphi_{n}^{S}-\varphi\right\|_{\infty}=O\left(h^{2}\right) .
$$

On the other hand, if $\alpha \geq 4$, then, from Theorem 4.2 and Theorem 4.6, we obtain

$$
\left\|\varphi_{n}^{M}-\varphi\right\|_{\infty}=O\left(h^{3}\right), \quad\left\|\widetilde{\varphi}_{n}^{M}-\varphi\right\|_{\infty}=O\left(h^{4}\right) .
$$

Thus, the sequence $\left\{\varphi_{n}^{M}\right\}$ converges faster to the exact solution $\varphi$ than does the sequence $\left\{\varphi_{n}^{S}\right\}$, and the sequence $\left\{\widetilde{\varphi}_{n}^{M}\right\}$ converges faster than does the sequence $\left\{\varphi_{n}^{M}\right\}$. The above orders of convergence are validated by numerical results in Table 1 .

Next let $r \geq 1$. If $\alpha \geq r+3$, then from (2.22) and (2.23), we obtain

$$
\left\|\varphi_{n}^{C}-\varphi\right\|_{\infty}=O\left(h^{r+1}\right), \quad\left\|\varphi_{n}^{S}-\varphi\right\|_{\infty}=O\left(h^{r+3}\right) .
$$

On the other hand, if $\alpha \geq r+1$, then from Theorems 4.2 and 4.6, we obtain

$$
\left\|\varphi_{n}^{M}-\varphi\right\|_{\infty}=O\left(h^{r+3}\right), \quad\left\|\widetilde{\varphi}_{n}^{M}-\varphi\right\|_{\infty}=O\left(h^{2 r+3}\right) .
$$

The above orders of convergence are validated for the case $r=1$ in Table 1.

Note that the improvement in the order of convergence in the iterated collocation method as compared to the collocation method is at most 2, irrespective of the value of $r$. On the other hand, the order of convergence $r+3$ in the modified projection method is improved to $2 r+3$ by one step of iteration.

5. Numerical results. We validate the convergence results that were obtained in Theorems 4.2 and 4.6 by the following numerical 
example from [4]. For comparison, the corresponding results for the collocation and the iterated collocation methods are also given.

Consider

$$
x(s)-\int_{0}^{1} \kappa(s, t)\left[f(t, x(t)] d t=\int_{0}^{1} \kappa(s, t) z(t) d t, \quad 0 \leq s \leq 1,\right.
$$

where

$$
\kappa(s, t)= \begin{cases}(1-s) t & 0 \leq t \leq s \leq 1 \\ s(1-t) & 0 \leq s \leq t \leq 1\end{cases}
$$

and

$$
f(t, u)=\frac{1}{1+t+u}
$$

with $z(t)$ so chosen that

$$
\varphi(t)=\frac{t(1-t)}{t+1}
$$

is the solution of (5.1).

In this example, $\alpha$ can be chosen as large as we want, and hence, $\beta=r+1$. Consider the following uniform partition of $[0,1]$ :

$$
\Delta: 0<\frac{1}{n}<\frac{2}{n}<\cdots<\frac{n}{n}=1 .
$$

5.1. Interpolation at mid-points: $r=0$. Let $\mathcal{P}_{0, \Delta}$ be the space of piecewise constant polynomials with respect to the partition (5.2). Let

$$
\tau^{j}=\frac{2 j-1}{2 n}, \quad 1 \leq j \leq n,
$$

and

$$
Q_{n}: C_{\Delta} \longrightarrow \mathcal{P}_{0, \Delta}
$$

be the interpolatory projection defined by $\left(Q_{n} x\right)\left(\tau^{j}\right)=x\left(\tau^{j}\right), 1 \leq j \leq$ $n$. Recall from (4.20) that the expected orders of convergence in the collocation and the iterated collocation methods are, respectively, 1 and 2. From (4.21), we see that the expected orders of convergence in the modified projection and the iterated modified projection methods are, respectively, 3 and 4 . 
TABLE 1

\begin{tabular}{|c|cc|cc|}
\hline$n$ & $\left\|\varphi-\varphi_{n}^{C}\right\|_{\infty}$ & $\delta_{C}$ & $\left\|\varphi-\varphi_{n}^{S}\right\|_{\infty}$ & $\delta_{S}$ \\
\hline 2 & $1.50 \times 10^{-1}$ & & $1.30 \times 10^{-3}$ & \\
4 & $9.54 \times 10^{-2}$ & 0.65 & $2.31 \times 10^{-4}$ & 2.49 \\
6 & $6.87 \times 10^{-2}$ & 0.81 & $1.02 \times 10^{-4}$ & 2.01 \\
8 & $5.34 \times 10^{-2}$ & 0.88 & $5.81 \times 10^{-5}$ & 1.95 \\
10 & $4.35 \times 10^{-2}$ & 0.92 & $3.67 \times 10^{-5}$ & 2.07 \\
12 & $3.66 \times 10^{-2}$ & 0.95 & $2.59 \times 10^{-5}$ & 1.90 \\
\hline \hline$n$ & $\left\|\varphi-\varphi_{n}^{M}\right\|_{\infty}$ & $\delta_{M}$ & $\left\|\varphi-\widetilde{\varphi}_{n}^{M}\right\|_{\infty}$ & $\delta_{I M}$ \\
\hline 2 & $1.31 \times 10^{-3}$ & & $1.31 \times 10^{-3}$ & \\
4 & $1.68 \times 10^{-4}$ & 2.97 & $7.77 \times 10^{-5}$ & 4.07 \\
6 & $5.71 \times 10^{-5}$ & 2.66 & $1.47 \times 10^{-5}$ & 4.10 \\
8 & $2.62 \times 10^{-5}$ & 2.71 & $4.76 \times 10^{-6}$ & 3.92 \\
10 & $1.37 \times 10^{-5}$ & 2.90 & $1.87 \times 10^{-6}$ & 4.19 \\
12 & $8.22 \times 10^{-6}$ & 2.80 & $9.52 \times 10^{-7}$ & 3.69 \\
\hline
\end{tabular}

TABLE 2

\begin{tabular}{|c|cc|cc|}
\hline$n$ & $\left\|\varphi-\varphi_{n}^{G}\right\|_{\infty}$ & $\delta_{G}$ & $\left\|\varphi-\varphi_{n}^{S}\right\|_{\infty}$ & $\delta_{S}$ \\
\hline 2 & $5.49 \times 10^{-2}$ & & $1.25 \times 10^{-3}$ & \\
4 & $1.58 \times 10^{-2}$ & 1.80 & $8.80 \times 10^{-5}$ & 3.82 \\
6 & $7.39 \times 10^{-3}$ & 1.87 & $1.79 \times 10^{-5}$ & 3.92 \\
8 & $4.23 \times 10^{-3}$ & 1.94 & $6.24 \times 10^{-6}$ & 3.66 \\
10 & $2.71 \times 10^{-3}$ & 2.00 & $2.27 \times 10^{-6}$ & 4.54 \\
\hline \hline$n$ & $\left\|\varphi-\varphi_{n}^{M}\right\|_{\infty}$ & $\delta_{M}$ & $\left\|\varphi-\widetilde{\varphi}_{n}^{M}\right\|_{\infty}$ & $\delta_{I M}$ \\
\hline 2 & $3.53 \times 10^{-4}$ & & $3.33 \times 10^{-4}$ & \\
4 & $1.36 \times 10^{-5}$ & 4.70 & $4.76 \times 10^{-6}$ & 6.12 \\
6 & $2.87 \times 10^{-6}$ & 3.83 & $4.22 \times 10^{-7}$ & 5.97 \\
8 & $1.00 \times 10^{-6}$ & 3.64 & $7.38 \times 10^{-8}$ & 6.06 \\
10 & $4.31 \times 10^{-7}$ & 3.79 & $1.87 \times 10^{-8}$ & 6.16 \\
\hline
\end{tabular}

The numerical quadrature in the computations needs to be so chosen as to preserve the above orders of convergence. As the kernel is only continuous, the order of convergence in the composite Gauss 2 point rule with respect to the partition (5.2) gets reduced from $h^{4}$ to $h^{2}$. Hence, in order to retain the order of convergence $h^{4}$, we choose the Gauss 2 point rule with respect to a uniform partition with $n^{2}$ subintervals. 
In Table $1, \delta_{C}, \delta_{S}, \delta_{M}$ and $\delta_{I M}$ denote the computed orders of convergence in the collocation, the iterated collocation, the modified projection and the iterated modified projection methods, respectively. It can be seen that the computed values of order of convergence match well with the theoretically predicted values.

5.2. Interpolation at Gauss 2 points: $r=1$. Let $\mathcal{P}_{1, \Delta}$ be the space of piecewise linear polynomials with respect to the partition (5.2). The Gauss 2 points in $[(j-1) / n, j / n]$ are given by

$$
\tau_{1}^{j}=\frac{2 j-1}{2 n}-\frac{1}{2 n} \frac{1}{\sqrt{3}}
$$

and

$$
\tau_{2}^{j}=\frac{2 j-1}{2 n}+\frac{1}{2 n} \frac{1}{\sqrt{3}}, \quad 1 \leq j \leq n .
$$

Let $Q_{n}: C_{\Delta} \rightarrow \mathcal{P}_{1, \Delta}$ be the interpolatory projection defined by

$$
\left(Q_{n} x\right)\left(\tau_{1}^{j}\right)=x\left(\tau_{1}^{j}\right), \quad\left(Q_{n} x\right)\left(\tau_{2}^{j}\right)=x\left(\tau_{2}^{j}\right), \quad 1 \leq j \leq n .
$$

Recall from equation (4.22) that the expected orders of convergence in the collocation and the iterated collocation methods are, respectively, 2 and 4. From (4.23), we see that the expected orders of convergence in the modified projection and the iterated modified projection methods are, respectively, 4 and 5 .

In the collocation and the iterated collocation methods, the Gauss 2 point rule with $n^{2}$ subintervals is chosen, whereas in the modified projection and the iterated modified projection methods, the Gauss 2 point rule with $n^{3}$ subintervals, which has the order of convergence $h^{6}$, is chosen.

It can be seen from Table 2 that computed orders of convergence in the collocation, the iterated collocation and the modified projection methods match with the theoretically predicted values. However, in the case of the iterated modified projection method, the computed order of convergence seems to be better than the theoretically predicted value.

6. Conclusion. We consider modified projection and iterated modified projection methods for approximate solutions of a Urysohn integral equation. The kernel of the integral operator is of the type of Green's 
function, and the projection is chosen to be an interpolatory projection at $r+1$ Gauss points. The main contribution of this paper is Theorem 4.6, in which the order of convergence of the iterated modified projection solution $\widetilde{\varphi}_{n}^{M}$ is obtained. This result shows that the sequence $\left\{\widetilde{\varphi}_{n}^{M}\right\}$ converges faster to the exact solution $\varphi$ than does the sequence $\left\{\varphi_{n}^{S}\right\}$ obtained in the iterated collocation method. It is to be noted that the size of the system of equations that must be solved in implementing the iterated modified projection remains the same as for the iterated collocation method.

Acknowledgments. We would like to thank Professor K.E. Atkinson and the anonymous referees for their comments which helped in improving the presentation of the paper.

\section{REFERENCES}

1. K. Atkinson, I. Graham and I. Sloan, Piecewise continuous collocation for integral equations, SIAM J. Numer. Anal. 20 (1983), 172-186.

2. K.E. Atkinson, A survey of numerical methods for solving nonlinear integral equations, J. Integral Equations Appl. 4 (1992), 15-46.

3. , The numerical solutions of integral equations of the second kind, Cambridge University Press, Cambridge, UK, 1997.

4. K.E. Atkinson and F.A. Potra, Projection and iterated projection methods for nonlinear integral equations, SIAM J. Numer. Anal. 24 (1987), 1352-1373.

5. F. Chatelin and R. Lebbar, Superconvergence results for the iterated projection method applied to a Fredholm integral equation of the second kind and the corresponding eigenvalue problem, J. Integral Equations Appl. 6 (1984), 71-91.

6. L. Grammont, A Galerkin's perturbation type method to approximate a fixed point of a compact operator, Int. J. Pure Appl. Math. 69 (2011), 1-14.

7. L. Grammont and R.P. Kulkarni, A superconvergent projection method for nonlinear compact operator equations, C.R. Acad. Sci. Paris 342 (2006), 215-218.

8. L. Grammont, R.P. Kulkarni and P. Vasconcelos, Modified projection and the iterated modified projection methods for nonlinear integral equations, J. Integral Equations Appl. 25 (2013), 481-516.

9. M.A. Krasnoselskii, Topological methods in the theory of nonlinear integral equations, Pergamon Press, London, 1964.

10. M.A. Krasnoselskii, G.M. Vainikko, P.P. Zabreiko, Ya.B. Rutitskii and V.Ya. Stetsenko, Approximate solution of operator equations, P. Noordhoff, Groningen, 1972 .

11. M.A. Krasnoselskii and P.P. Zabreiko, Geometrical methods of nonlinear analysis, Springer-Verlag, Berlin, 1984. 
12. R.P. Kulkarni, A superconvergence result for solutions of compact operator equations, Bull. Austral. Math. Soc. 68 (2003), 517-528.

13. On improvement of the iterated Galerkin solution of the second kind integral equations, J. Numer. Math. 13 (2005), 205-218.

14. R.P. Kulkarni and T.J. Nidhin, Asymptotic error analysis of projection and modified projection methods for nonlinear integral equations, J. Integral Equations Appl. 27 (2015), 67-101.

15. I. Sloan, Improvement by iteration for compact operator equations, Math. Comp. 30, 758-764.

16. G.M. Vainikko, Galerkin's perturbation method and general theory of approximate methods for non-linear equations, USSR Comput. Math. Math. Phys. 7 (1967), 1-41.

Department of Mathematics, I.I.T. Bombay, Powai, Mumbai 400076, India Email address: rpk@math.iitb.ac.in

Department of Mathematics, I.I.T. Bombay, Powai, Mumbai 400076, India Email address: nidhin@math.iitb.ac.in 